This is a self-archived version of an original article. This version may differ from the original in pagination and typographic details.

Author(s): Xu, Haiqing

Title: Weighted estimates for diffeomorphic extensions of homeomorphisms

Year: 2020

Version: Accepted version (Final draft)

Copyright: @ 2021 EMS Publishing House

Rights: In Copyright

Rights url: http://rightsstatements.org/page/lnC/1.0/?language=en

Please cite the original version:

$\mathrm{Xu}, \mathrm{H}$. (2020). Weighted estimates for diffeomorphic extensions of homeomorphisms.

Rendiconti Lincei: Matematica e Applicazioni, 31(1), 151-189. https://doi.org/10.4171/RLM/884 


\title{
Weighted estimates for diffeomorphic extensions of homeomorphisms
}

\author{
Haiqing $\mathrm{Xu}^{1,2}$ \\ ${ }^{1}$ Department of Mathematics and Statistics, University of \\ Jyväskylä, PO Box 35, FI-40014 Jyväskylä, Finland \\ ${ }^{2}$ School of Mathematical Sciences, University of Science and \\ Technology of China, Hefei 230026, P. R. China \\ E-mail address: hqxu@mail.ustc.edu.cn
}

\begin{abstract}
Let $\Omega \subset \mathbb{R}^{2}$ be an internal chord-arc domain and $\varphi: \mathbb{S}^{1} \rightarrow \partial \Omega$ be a homeomorphism. Then there is a diffeomorphic extension $h: \mathbb{D} \rightarrow \Omega$ of $\varphi$. We study the relationship between weighted integrability of the derivatives of $h$ and double integrals of $\varphi$ and of $\varphi^{-1}$.
\end{abstract}

Keywords: Poisson extension, diffeomorphism, internal chord-arc domain.

\section{Introduction}

Let $\Omega \subset \mathbb{R}^{2}$ be a bounded convex domain. Suppose that $\varphi$ is a homeomorphism from the unit circle $\mathbb{S}^{1}$ onto $\partial \Omega$. Then, by Radó [13], Kneser [7], Choquet [3] and Lewy [10], the complex-valued Poisson extension $h$ of $\varphi$ is a diffeomorphism from $\mathbb{D}$ onto $\Omega$. We are interested in the integrability degrees of the derivatives of $h$. In 2007, G. C. Verchota [14] proved that the derivatives of $h$ may fail to be square integrable but that they are necessarily $p$-integrable over $\mathbb{D}$ for all $p<2$. In 2009, T. Iwaniec, G. J. Martin and C. Sbordone improved on [5] by showing that the derivatives belong to weak- $L^{2}$ with sharp estimates. Actually

$$
\int_{\mathbb{D}}|D h(z)|^{2} d z \approx \int_{\mathbb{S}^{1}} \int_{\mathbb{S}^{1}} \frac{|\varphi(\xi)-\varphi(\eta)|^{2}}{|\xi-\eta|^{2}}|d \xi||d \eta|
$$


since harmonic functions minimize the $L^{2}$-energy and the right-hand side of (1.1) is the trace norm of $\dot{W}^{1,2}(\mathbb{D})$. In [1], it was further shown that if additionally $\partial \Omega$ is a $C^{1}$-regular Jordan curve then

$$
\int_{\mathbb{D}}|D h(z)|^{2} d z<\infty \Leftrightarrow \int_{\partial \Omega} \int_{\partial \Omega}|\log | \varphi^{-1}(\xi)-\varphi^{-1}(\eta)|||d \xi||d \eta|<\infty .
$$

All the above results require the target domain to be convex.

If $\Omega$ is a bounded non-convex Jordan domain, then there exists a homeomorphism $\varphi: \mathbb{S}^{1} \rightarrow \partial \Omega$ for which the harmonic extension fails to map $\mathbb{D}$ homeomorphically onto $\Omega$, see $[3,7]$. Hence we cannot use the harmonic extension to produce a diffeomorphic extension. Nevertheless, (weighted) analogs of the results as (1.2) for diffeomorphic extensions in the case of an internal chord-arc Jordan domain exist, see [9]. For the definition of (internal) chord-arc domains, we refer to Definition 2.1. Notice that each bounded convex Jordan domain is a chord-arc domain. In this paper, we generalize the results in [9] to the weighted $L^{p}$-setting.

Let $\Omega$ be an internal chord-arc Jordan domain with the internal distance $\lambda_{\Omega}$. Assume that $h: \mathbb{D} \rightarrow \Omega$ is a diffeomorphism and $\varphi: \mathbb{S}^{1} \rightarrow \partial \Omega$ is a homeomorphism. Set $\delta(z)=1-|z|$. Given $p>1, \alpha \in \mathbb{R}, \lambda \in \mathbb{R}$, we define

$$
\begin{gathered}
I_{1}(p, \alpha, \lambda, h)=\int_{\mathbb{D}}|D h(z)|^{p} \delta^{\alpha}(z) \log ^{\lambda}\left(2 \delta^{-1}(z)\right) d z \\
I_{2}(p, \alpha, \lambda, h)=\int_{\mathbb{D}}|D h(z)|^{p} \log ^{\lambda}(e+|D h(z)|) \delta^{\alpha}(z) d z \\
\mathcal{U}(p, \alpha, \lambda, \varphi)=\int_{\mathbb{S}^{1}} \int_{\mathbb{S}^{1}} \frac{\lambda_{\Omega}^{p}(\varphi(\xi), \varphi(\eta))}{|\xi-\eta|^{p-\alpha}} \log ^{\lambda}\left(e+\frac{\lambda_{\Omega}(\varphi(\xi), \varphi(\eta))}{|\xi-\eta|}\right)|d \eta||d \xi|, \\
\mathcal{A}_{p, \alpha, \lambda}(t)=\int_{1}^{t}-x^{1+\alpha-p} \log _{2}^{\lambda}\left(x^{-1}\right) d x \quad \forall t \geq 0, \\
\mathcal{V}(p, \alpha, \lambda, \varphi)=\int_{\partial \Omega}\left(\int_{\partial \Omega} \mathcal{A}_{p, \alpha, \lambda}\left(\left|\varphi^{-1}(\xi)-\varphi^{-1}(\eta)\right|\right)|d \eta|\right)^{p-1}|d \xi| .
\end{gathered}
$$

Our main result is the following theorem.

Theorem 1.1. Let $\Omega \subset \mathbb{R}^{2}$ be an internal chord-arc Jordan domain and $\varphi: \mathbb{S}^{1} \rightarrow$ $\partial \Omega$ be a homeomorphism. There is a diffeomorphic extension $h: \mathbb{D} \rightarrow \Omega$ of $\varphi$ for which, for any $p>1$, we have that

(1) if either $\alpha \in(p-2,+\infty)$ and $\lambda \in \mathbb{R}$ or $\alpha=p-2$ and $\lambda \in(-\infty,-1)$, both $I_{1}(p, \alpha, \lambda, h)$ and $I_{2}(p, \alpha, \lambda, h)$ are finite. 
(2) if either $\alpha \in(-1, p-2)$ and $\lambda \in \mathbb{R}$ or $\alpha=p-2$ and $\lambda \in[-1,+\infty)$,

both $I_{1}(p, \alpha, \lambda, h)$ and $I_{2}(p, \alpha, \lambda, h)$ are comparable to $\mathcal{U}(p, \alpha, \lambda, \varphi)$.

Moreover whenever $p \in(1,2]$

both $I_{1}(p, \alpha, \lambda, h)$ and $I_{2}(p, \alpha, \lambda, h)$ dominate $\mathcal{V}(p, \alpha, \lambda, \varphi)$,

while

$\mathcal{V}(p, \alpha, \lambda, \varphi)$ controls both $I_{1}(p, \alpha, \lambda, h)$ and $I_{2}(p, \alpha, \lambda, h)$

for all $p \in[2,+\infty)$. Furthermore both $I_{1}(p, \alpha, \lambda, h)$ and $I_{2}(p, \alpha, \lambda, h)$ are in general comparable to $\mathcal{V}(p, \alpha, \lambda, \varphi)$ only for $p=2$.

For any $p>1$, there is no diffeomorphic extension $h: \mathbb{D} \rightarrow \Omega$ of $\varphi$ for which $I_{1}(p, \alpha, \lambda, h)<+\infty$ for either $\alpha \in(-\infty,-1)$ and $\lambda \in \mathbb{R}$ or $\alpha=-1$ and $\lambda \in$ $[-1,+\infty)$; or for which $I_{2}(p, \alpha, \lambda, h)<+\infty$ for some $\alpha \in(-\infty,-1]$ and $\lambda \in \mathbb{R}$.

Motivated by (1.2), one could hope to use $\mathcal{V}(p, \alpha, \lambda, \varphi)$ to control both $I_{1}(p, \alpha, \lambda, h)$ and $I_{2}(p, \alpha, \lambda, h)$. Example 4.2 together with Example 4.3 shows that $\mathcal{V}(p, \alpha, \lambda, \varphi)$ is comparable to $I_{1}(p, \alpha, \lambda, h)$ or $I_{2}(p, \alpha, \lambda, h)$ only when $p=2$. Theorem 1.1 does not cover the case where $p>1, \alpha=-1$ and $\lambda \in(-\infty,-1)$. We will return to this case in a future paper.

The structure of this paper is the following. In the next section, we give some preliminaries. Section 3 is the proof of Theorem 1.1. The final section contains several examples related to Theorem 1.1 (2).

\section{Preliminaries}

By $s \gg 1$ and $t \ll 1$ we mean that $s$ is sufficiently large and $t$ is sufficiently small, respectively. By $f \lesssim g$ we mean that there exists a constant $C>0$ such that $f(x) \leq C g(x)$ for every $x$. If $f \lesssim g$ and $g \lesssim f$ we may denote $f \approx g$. By $\mathbb{N}$ and $\mathbb{R}$ we denote the set of all positive integers and the set of all real numbers. Let $\mathcal{L}^{2}$ (respectively $\mathcal{L}^{1}$ ) be the 2-dimensional (1-dimensional) Lebesgue measure. For sets $E \in \mathbb{R}^{2}$ and $F \in \mathbb{R}^{2}$, let diam $(E)$ be the diameter of $E$, and dist $(E, F)$ be the Euclidean distance between $E$ and $F$. Let $B(p, r)$ be the disk with center $P$ and radius $r$.

Definition 2.1. A Jordan domain $\Omega \subset \mathbb{R}^{2}$ is an internal chord-arc Jordan domain if $\partial \Omega$ is rectifiable and there is a constant $C>0$ such that for all $w_{1}, w_{2} \in \partial \Omega$,

$$
\ell\left(w_{1}, w_{2}\right) \leq C \lambda_{\Omega}\left(w_{1}, w_{2}\right)
$$


where $\ell\left(w_{1}, w_{2}\right)$ is the arc length of the shorter arc of $\partial \Omega$ joining $w_{1}$ to $w_{2}$, and $\lambda_{\Omega}\left(w_{1}, w_{2}\right)$ is the internal distance between $w_{1}, w_{2}$, which is defined as

$$
\lambda_{\Omega}\left(w_{1}, w_{2}\right)=\inf _{\alpha} \ell(\alpha)
$$

where the infimum is taken over all rectifiable $\operatorname{arcs} \alpha \subset \Omega$ joining $w_{1}$ and $w_{2}$; if there is no rectifiable curve joining $w_{1}$ and $w_{2}$, we set $\lambda_{\Omega}\left(w_{1}, w_{2}\right)=\infty$; cf. [12, Section 3.1] or [2, Section 2].

If (2.1) holds for the Euclidean distance instead of the internal distance, we call $\Omega$ be a chord-arc domain. Naturally, every chord-arc Jordan domain is an internal chord-arc domain, but there are internal chord-arc domains that fail to be chord-arc; e.g. the standard cardioid domain

$$
\Delta=\left\{(x, y) \in \mathbb{R}^{2}:\left(x^{2}+y^{2}\right)^{2}-4 x\left(x^{2}+y^{2}\right)-4 y^{2}<0\right\} .
$$

\subsection{Dyadic decomposition}

Given $j \in \mathbb{N}$ and $k=1, \ldots, 2^{j}$, let

$$
I_{j, k}=\left[2 \pi(k-1) 2^{-j}, 2 \pi k 2^{-j}\right], \Gamma_{j, k}=\left\{e^{i \theta}: \theta \in I_{j, k}\right\} .
$$

Then $\left\{I_{j, k}\right\}$ is a dyadic decomposition of $[0,2 \pi]$ and $\left\{\Gamma_{j, k}\right\}$ is a dyadic decomposition of $\mathbb{S}^{1}$. We call $\Gamma_{j, k}$ a $j$-level dyadic arc. Moreover we have that

$$
\ell\left(\Gamma_{j, k}\right) \approx 2^{-j} \quad \forall j \in \mathbb{N} \text { and } k=1, \ldots, 2^{j} .
$$

Based on (2.2), there is a decomposition of the unit disk $\mathbb{D}$ given by $\left\{Q_{j, k}: j \in\right.$ $\mathbb{N}$ and $\left.k=1, \ldots, 2^{j}\right\}$, where

$$
Q_{j, k}=\left\{r e^{i \theta}: 1-2^{1-j} \leq r \leq 1-2^{-j} \text { and } \theta \in I_{j, k}\right\} .
$$

By (2.3) it follows that

$$
\mathcal{L}^{2}\left(Q_{j, k}\right) \approx 2^{-2 j} \approx \ell\left(\Gamma_{j, k}\right)^{2} \quad \forall j \in \mathbb{N} \text { and } k=1, \ldots, 2^{j}
$$

Moreover there is a uniform constant $C>0$ such that for any $Q_{j, k}$ there is a disk $B_{j, k}$ satisfying

$$
B_{j, k} \subset Q_{j, k} \subset C B_{j, k}
$$




\section{$2.2 \quad A_{p}$ weights}

Definition 2.2. For a given $p \in(1,+\infty)$, a locally integrable function $w: \mathbb{R}^{2} \rightarrow$ $[0,+\infty)$ is an $A_{p}$ weight if there is a constant $C>0$ such that for any disk $B \subset \mathbb{R}^{2}$ we have that

$$
\frac{1}{\mathcal{L}^{2}(B)} \int_{B} w(x) d x \leq C\left(\frac{1}{\mathcal{L}^{2}(B)} \int_{B} w(x)^{\frac{1}{1-p}} d x\right)^{1-p}
$$

Next, $w$ is an $A_{1}$ weight if there is a constant $C>0$ such that

$$
\frac{1}{\mathcal{L}^{2}(B)} \int_{B} w(z) d z \leq C w(x)
$$

for each disk $B \subset \mathbb{R}^{2}$ and all $x \in B$.

For more information on $A_{p}$ weights, we recommend $[4,6,11]$. Let $\delta(x)=$ $\operatorname{dist}\left(\mathbb{S}^{1}, x\right)$. Given $\alpha \in(-1, p-1)$ and $\lambda \in \mathbb{R}$, we define

$$
w_{\alpha, \lambda}(x)= \begin{cases}\delta(x)^{\alpha} \log ^{\lambda}\left(2 \delta^{-1}(x)\right) & 0 \leq|x| \leq 2, \\ \log ^{\lambda}(2) & |x| \geq 2\end{cases}
$$

It is well known that $w_{\alpha, 0}$ belongs to $A_{p}$. We now generalize this to all $\lambda \in \mathbb{R}$.

Proposition 2.3. Let $p \geq 1$ and $w_{\alpha, \lambda}$ be as in (2.7). Then $w_{\alpha, \lambda}$ is an $A_{p}$ weight for all $\alpha \in(-1, p-1)$ and $\lambda \in \mathbb{R}$.

Proof. The idea of proof is to use the Jones factorization of $A_{p}$ weights (see [6]), i.e. we should prove $w_{\alpha, \lambda}=w_{1} w_{2}^{1-p}$ for two $A_{1}$ weights $w_{1}$ and $w_{2}$.

We first consider the case $\lambda \geq 0$. For a given $\alpha \in(-1, p-1)$, there uniquely exist $a_{1} \in(0,1)$ and $a_{2} \in(0,1)$ such that $\alpha=a_{1}(-1)+a_{2}(p-1)$. Set $\alpha_{1}=-a_{1}$, $\alpha_{2}=-a_{2}, \lambda_{1}=p \lambda$ and $\lambda_{2}=\lambda$. We define

$$
w_{1}(x)= \begin{cases}\delta(x)^{\alpha_{1}} \log ^{\lambda_{1}}\left(2 \delta^{-1}(x)\right) & 0 \leq|x| \leq 2, \\ \log ^{\lambda_{1}}(2) & |x| \geq 2\end{cases}
$$

and

$$
w_{2}(x)= \begin{cases}\delta(x)^{\alpha_{2}} \log ^{\lambda_{2}}\left(2 \delta^{-1}(x)\right) & 0 \leq|x| \leq 2, \\ \log ^{\lambda_{2}}(2) & |x| \geq 2\end{cases}
$$

We next prove that $w_{1}$ is an $A_{1}$ weight, i.e.

$$
f_{B} w_{1}(x) d x \lesssim \inf _{x \in B} w_{1}(x)
$$


for every disk $B \subset \mathbb{R}^{2}$. Let $d_{B}=\operatorname{dist}\left(B, \mathbb{S}^{1}\right)$.

Case 1: $d_{B} \geq \operatorname{diam}(B) / 2$. We have that

$$
d_{B} \leq \delta(x) \leq 3 d_{B} \quad \forall x \in B .
$$

If $1 \leq d_{B}$, then $\delta(x) \geq 1$ for all $x \in B$. Therefore $w_{1}(x)=\log ^{\lambda_{1}}(2)$ whenever $x \in$ $B$. Of course (2.10) holds now. If $3 d_{B} \leq 1$, then $w_{1}(x)=\delta(x)^{\alpha_{1}} \log ^{\lambda_{1}}\left(2 \delta^{-1}(x)\right)$ for all $x \in B$. By $(2.11)$ it hence follows that $w_{1}(x) \approx d_{B}^{\alpha_{1}} \log ^{\lambda_{1}}\left(2 d_{B}^{-1}\right)$ whenever $x \in B$. Therefore (2.10) holds. If $d_{B}<1<3 d_{B}$, let $B_{1}=\left\{x \in B: d_{B}<\delta(x)<\right.$ $1\}$ and $B_{2}=\left\{x \in B: 1 \leq \delta(x)<3 d_{B}\right\}$. Then $B=B_{1} \cup B_{2}$ and

$$
w_{1}(x)=\log ^{\lambda_{1}}(2) \quad \text { whenever } x \in B_{2} \text {. }
$$

Since

$$
\left[t^{\alpha_{1}} \log ^{\lambda_{1}}\left(2 t^{-1}\right)\right]^{\prime}=t^{\alpha_{1}-1} \log ^{\lambda_{1}}\left(2 t^{-1}\right)\left(\alpha_{1}-\frac{\lambda_{1}}{\log \left(2 t^{-1}\right)}\right)<0,
$$

for all $t \in(0,1]$, we have that

$$
w_{1}(x) \leq d_{B}^{\alpha_{1}} \log ^{\lambda_{1}}\left(2 d_{B}^{-1}\right) \leq \frac{\log ^{\lambda_{1}}(6)}{3^{\alpha_{1}}} \quad \forall x \in B_{1} .
$$

Combining (2.12) and (2.14) implies that

$$
\begin{aligned}
f_{B} w_{1}(x) d x & =\frac{1}{\mathcal{L}^{2}(B)}\left(\int_{B_{1}} w_{1}+\int_{B_{2}} w_{1}\right) \\
& \leq \frac{1}{\mathcal{L}^{2}(B)}\left(\mathcal{L}^{2}\left(B_{1}\right) \frac{\log ^{\lambda_{1}}(6)}{3^{\alpha_{1}}}+\mathcal{L}^{2}\left(B_{2}\right) \log ^{\lambda_{1}}(2)\right) \\
& \lesssim \log ^{\lambda_{1}}(2)=\inf _{x \in B} w_{1}(x) .
\end{aligned}
$$

Case 2: $d_{B}<\operatorname{diam}(B) / 2$ and $\operatorname{diam}(B) \leq 2 / 3$. Pick $x^{\prime} \in \partial B$ and $x_{0} \in \mathbb{S}^{1}$ such that $\operatorname{dist}\left(B, \mathbb{S}^{1}\right)=\left|x^{\prime}-x_{0}\right|$. Let $r_{B}=3 \operatorname{diam}(B) / 2$. Since

$$
\left|x-x_{0}\right| \leq\left|x-x^{\prime}\right|+\left|x^{\prime}-x_{0}\right| \leq r_{B}
$$

for all $x \in B$, we have $B \subset B\left(x_{0}, r_{B}\right)$. Let $E=\left\{x \in \mathbb{R}^{2}: \operatorname{dist}\left(x, \mathbb{S}^{1}\right)<r_{B}\right\}$. Then $B\left(x_{0}, r_{B}\right) \subset E$. Since $\mathcal{L}^{2}\left(B\left(x_{0}, r_{B}\right)\right)=\pi r_{B}^{2}$ and $\mathcal{L}^{2}(E)=4 \pi r_{B}$, the maximal number of pairwise disjoint open disks $B\left(x, r_{B}\right)$ with $x \in \mathbb{S}^{1}$ is less than $4 r_{B}^{-1}$. We have that

$$
\begin{aligned}
\frac{1}{\mathcal{L}^{2}(B)} \int_{B} w_{1}(x) d x & \leq \frac{1}{\mathcal{L}^{2}(B)} \int_{B\left(x_{0}, r_{B}\right)} w_{1}(x) d x \\
& \lesssim \frac{r_{B}}{\mathcal{L}^{2}(B)} \int_{E} w_{1}(x) d x \approx \frac{1}{r_{B}} \int_{0}^{r_{B}} t^{\alpha_{1}} \log ^{\lambda_{1}}\left(2 t^{-1}\right) d t .
\end{aligned}
$$


Notice that

$$
\left[t^{\alpha_{1}+1} \log ^{\lambda_{1}}\left(2 t^{-1}\right)\right]^{\prime}=t^{\alpha_{1}} \log ^{\lambda_{1}}\left(2 t^{-1}\right)\left(\alpha_{1}+1-\frac{\lambda_{1}}{\log \left(2 t^{-1}\right)}\right) \quad t>0 .
$$

Since $\lim _{t \rightarrow 0^{+}} \alpha_{1}+1-\frac{\lambda_{1}}{\log \left(2 t^{-1}\right)}=\alpha_{1}+1$ and $\alpha_{1}+1-\frac{\lambda_{1}}{\log \left(2 t^{-1}\right)}$ is decreasing with respect to $t>0$, there exists $\epsilon \in(0,1)$ determined by $\alpha_{1}$ and $\lambda_{1}$ such that $\alpha_{1}+1-\frac{\lambda_{1}}{\log \left(2 \epsilon^{-1}\right)} \geq\left(\alpha_{1}+1\right) / 2$. We then obtain from (2.16) that

$$
\left[t^{\alpha_{1}+1} \log ^{\lambda_{1}}\left(2 t^{-1}\right)\right]^{\prime} \geq \frac{\alpha_{1}+1}{2} t^{\alpha_{1}} \log ^{\lambda_{1}}\left(2 t^{-1}\right)
$$

for all $t \in\left[0, \epsilon r_{B}\right]$. Therefore

$$
\int_{0}^{\epsilon r_{B}} t^{\alpha_{1}} \log ^{\lambda_{1}}\left(2 t^{-1}\right) d t=\frac{2\left(\epsilon r_{B}\right)^{\alpha_{1}+1}}{\alpha_{1}+1} \log ^{\lambda_{1}}\left(2\left(\epsilon r_{B}\right)^{-1}\right) \lesssim r_{B}^{\alpha_{1}+1} \log ^{\lambda_{1}}\left(2 r_{B}^{-1}\right)
$$

Moreover by (2.13) we have that

$$
\int_{\epsilon r_{B}}^{r_{B}} t^{\alpha_{1}} \log ^{\lambda_{1}}\left(2 t^{-1}\right) d t \leq\left(r_{B}-\epsilon r_{B}\right)\left(\epsilon r_{B}\right)^{\alpha_{1}} \log ^{\lambda_{1}}\left(2\left(\epsilon r_{B}\right)^{-1}\right) \lesssim r_{B}^{\alpha_{1}+1} \log ^{\lambda_{1}}\left(2 r_{B}^{-1}\right) .
$$

Combining (2.15), (2.17) with (2.18) implies that

$$
\frac{1}{|B|} \int_{B} w_{1}(x) d x \lesssim r_{B}^{\alpha_{1}} \log ^{\lambda_{1}}\left(2 r_{B}^{-1}\right) \text {. }
$$

Together with

$$
r_{B}^{\alpha_{1}} \log ^{\lambda_{1}}\left(2 r_{B}^{-1}\right)=\inf _{t \in\left[0, r_{B}\right]} t^{\alpha_{1}} \log ^{\lambda_{1}}\left(2 t^{-1}\right)=\inf _{x \in E} w_{1}(x) \leq \inf _{x \in B} w_{1}(x),
$$

we hence obtain (2.10).

Case 3: $d_{B}<\operatorname{diam}(B) / 2$ and $\operatorname{diam}(B)>2 / 3$. Let $x^{\prime}$ and $x_{0}$ be as in Case 2. Then $\left|x^{\prime}\right|=1+\operatorname{dist}\left(x^{\prime}, \mathbb{S}^{1}\right) \leq 1+\operatorname{diam}(B) 2^{-1}$. Together with the fact that $\left|x-x^{\prime}\right| \leq \operatorname{diam}(B)$ for all $x \in B$, we have $B \subset B\left(0,1+r_{B}\right)$. Moreover by $(2.17)$ and (2.18), we obtain that

$$
\int_{B(0,2)} w_{1}(x) d x=\int_{B(0,1)}+\int_{B(0,2) \backslash B(0,1)}=4 \pi \int_{0}^{1} t^{\alpha_{1}} \log ^{\lambda_{1}}\left(2 t^{-1}\right) d t \approx 1 .
$$

Therefore

$$
\begin{aligned}
\frac{1}{\mathcal{L}^{2}(B)} \int_{B} w_{1}(x) d x & \lesssim \frac{1}{r_{B}^{2}}\left(\int_{B(0,2)}+\int_{B\left(0,1+r_{B}\right) \backslash B(0,2)}\right) \\
& \lesssim \frac{1}{r_{B}^{2}}\left(\mathcal{L}^{2}(B(0,2))+\log ^{\lambda_{1}}(2) \mathcal{L}^{2}\left(B\left(0,1+r_{B}\right) \backslash B(0,2)\right)\right) \\
& \lesssim 1 .
\end{aligned}
$$


Moreover by the monotonicity of $t^{\alpha_{1}} \log ^{\lambda_{1}}\left(2 t^{-1}\right)$ on $(0,+\infty)$, we have that

$$
\log ^{\lambda_{1}}(2)=\inf _{t \in\left[0,1+r_{B}\right]} t^{\alpha_{1}} \log ^{\lambda_{1}}\left(2 t^{-1}\right)=\inf _{x \in B\left(0,1+r_{B}\right)} w_{1}(x) \leq \inf _{x \in B} w_{1}(x) .
$$

By combining (2.19) with (2.20), we obtain (2.10).

By the analogous arguments as for (2.10), we obtain $w_{2} \in A_{1}$. Therefore the Jones factorization theorem implies that $w_{\alpha, \lambda} \in A_{p}$ for all $\alpha \in(-1, p-1)$ and $\lambda \geq 0$.

When $\lambda<0$, define $w_{1}$ and $w_{2}$ as in (2.8) and (2.9) with $\lambda_{1}=-\lambda, \lambda_{2}=$ $2 \lambda(1-p)^{-1}$ and both $\alpha_{1}$ and $\alpha_{2}$ invariant. By the same arguments as for the case $\lambda \geq 0$, we obtain that $w_{\alpha, \lambda} \in A_{p}$ whenever $\alpha \in(-1, p-1)$ and $\lambda<0$.

\subsection{A class of functions}

We define the Hardy-Littlewood maximal function for a Lebesgue measurable function $f$ in $\mathbb{R}^{2}$ as

$$
M_{f}(x)=\sup _{x \in B} f_{B}|f(z)| d z=\sup _{x \in B} \frac{1}{|B|} \int_{B}|f(z)| d z
$$

where the supremum is taken over all disks $B \subset \mathbb{R}^{2}$ containing $x$. Let $p \in(1, \infty)$ and $w$ be a weight. It is well-known that

$$
\int_{\mathbb{R}^{2}}\left|M_{f}(x)\right|^{p} w(x) d x \lesssim \int_{\mathbb{R}^{2}}|f(x)|^{p} w(x) d x
$$

if and only if $w$ is an $A_{p}$ weight. We generalize this to weighted Orlicz spaces. We begin with some definitions.

Definition 2.4. Let $\mathcal{F}$ be the collection of $\Phi:[0, \infty) \rightarrow[0, \infty)$, which is increasing and satisfies $\lim _{t \rightarrow 0} \Phi(t)=0$ and $\lim _{t \rightarrow \infty} \Phi(t)=\infty$. We say that $\Phi \in \mathcal{F}$ is the Young function, if $\Phi$ is convex on $[0, \infty)$ and $\lim _{t \rightarrow 0} \Phi(t) / t=\lim _{t \rightarrow \infty} t / \Phi(t)=0$.

Definition 2.5. We say that a function $\Phi:[0,+\infty) \rightarrow[0,+\infty)$ satisfies the $\Delta_{2}$-condition if there is a constant $C>0$ such that

$$
\Phi(2 t) \leq C \Phi(t)
$$

for all $t \in[0,+\infty)$.

Let $\Phi \in \mathcal{F}$ satisfying the $\Delta_{2}$-condition. Put

$$
h_{\Phi}(s)=\sup _{t>0} \frac{\Phi(s t)}{\Phi(t)} \quad s>0 .
$$


We define the lower index of $\Phi$ by

$$
i(\Phi)=\lim _{s \rightarrow 0} \frac{\log h_{\Phi}(s)}{\log s}=\sup _{0<s<1} \frac{\log h_{\Phi}(s)}{\log s} .
$$

The quantity $i(\Phi)$ is well defined, see [8]. The following lemma is from [8, Theorem 2.1.1].

Lemma 2.6. Let $\Phi$ be a Young function satisfying the $\Delta_{2}$-condition and $w$ be a weight on $\mathbb{R}^{2}$. Then the following conditions are equivalent:

1. $\int_{\mathbb{R}^{2}} \Phi\left(M_{f}(x)\right) w(x) d x \lesssim \int_{\mathbb{R}^{2}} \Phi(|f(x)|) w(x) d x$,

2. $w \in A_{i(\Phi)}$.

We next consider a special class of Young functions. Given $p>1$ and $\lambda \in \mathbb{R}$, we set

$$
\Phi_{p, \lambda}(t)=t^{p} \log ^{\lambda}(e+t) \quad \text { for } t \in[0,+\infty) .
$$

Proposition 2.7. Let $\Phi_{p, \lambda}$ be as in (2.21) with $p>1$ and $\lambda \geq 0$. Then $\Phi_{p, \lambda}$ is a Young function and satisfies the $\Delta_{2}$-condition on $[0, \infty)$. Moreover $i\left(\Phi_{p, \lambda}\right)=p$.

Proof. Simple calculations show that

$$
\Phi_{p, \lambda}^{\prime}(t)=\left(p \log (e+t)+\lambda \frac{t}{e+t}\right) t^{p-1} \log ^{\lambda-1}(e+t)
$$

and

$$
\begin{aligned}
\Phi_{p, \lambda}^{\prime \prime}(t)= & \left(p(p-1) \log ^{2}(e+t)+\lambda e \frac{t}{(e+t)^{2}} \log (e+t)+R_{p, \lambda}(t)\right) \\
& \times t^{p-2} \log ^{\lambda-2}(e+t)
\end{aligned}
$$

where $R_{p, \lambda}(t)=\lambda(\lambda-1)\left(t(e+t)^{-1}\right)^{2}+\lambda(2 p-1) t(e+t)^{-1} \log (e+t)$. Since $p \log (e+t)+\lambda t(e+t)^{-1}>0$ for all $t \in(0,+\infty)$, it follows from (2.22) that $\Phi_{p, \lambda}^{\prime}(t)>0$ for all $t>0$. Therefore $\Phi_{p, \lambda}$ is strictly increasing on $[0, \infty)$. If $\lambda \geq 1$, we have that

$$
R_{p, \lambda}(t) \geq 0 \quad \text { for all } t \geq 0 .
$$

Whenever $0 \leq \lambda<1$, since $t /(e+t)<1$ and $\log (e+t) \geq 1$ for all $t \geq 0$ we have that

$$
\begin{aligned}
R_{p, \lambda}(t) & =\frac{t}{e+t}\left(\lambda(\lambda-1) \frac{t}{e+t}+\lambda(2 p-1) \log (e+t)\right) \\
& \geq \frac{t}{e+t}(\lambda(\lambda-1)+\lambda(2 p-1))=\frac{t}{e+t}\left(\lambda^{2}+2 \lambda(p-1)\right) \geq 0
\end{aligned}
$$


for all $t \geq 0$. By (2.23), (2.24) and (2.25), we have that $\Phi_{p, \lambda}^{\prime \prime}(t) \geq 0$ for all $t \geq 0$. Therefore $\Phi_{p, \lambda}$ is convex on $[0,+\infty)$. Hence $\Phi_{p, \lambda}$ is a Young function whenever $p>1$ and $\lambda \geq 0$.

Since both $t^{p}$ and $\log ^{\lambda}(e+t)$ satisfy the $\Delta_{2}$-condition on $[0,+\infty), \Phi_{p, \lambda}$ satisfies $\Delta_{2}$-condition also. Since $h_{\Phi_{p, \lambda}}(s)=s^{p}$ whenever $s \in(0,1)$, we have $i\left(\Phi_{p, \lambda}\right)=$ $p$.

Remark 2.8. For $p>1$, let $\Phi_{p, \lambda}$ be as in (2.21) with $\lambda \geq 0$. Assume that $w$ is an $A_{p}$ weight. Given a Lebesgue measurable function $f$, by Lemma 2.6 and Proposition 2.7 we have that

$$
\int_{\mathbb{R}^{2}} \Phi_{p, \lambda}\left(M_{f}(x)\right) w(x) d x \lesssim \int_{\mathbb{R}^{2}} \Phi_{p, \lambda}(|f(x)|) w(x) d x .
$$

Let $\Phi_{p, \lambda}$ be as in (2.21) with $p>1$ and $\lambda<0$. By (2.22) and (2.23), we have that both monotonicity and convexity of $\Phi_{p, \lambda}$ may fail whenever $t \ll 1$, but still hold for all $t \gg 1$. We modify $\Phi_{p, \lambda}$ in a neighborhood of the origin such that it satisfies all conclusions in Proposition 2.7.

Since $2^{-1}(p+1) \log (e+t) \leq p \log (e+t)+\lambda t(e+t)^{-1}$ whenever $t \gg 1$, by (2.22) there is a constant $t_{2} \gg 1$ such that

$$
\frac{(p+1) \Phi_{p, \lambda}(t)}{2 t}=\frac{p+1}{2} t^{p-1} \log ^{\lambda}(e+t) \leq \Phi_{p, \lambda}^{\prime}(t)
$$

for all $t \geq t_{2}$. Without loss of generality, we assume that $\Phi_{p, \lambda}$ is strictly increasing and convex on $\left[t_{2}, \infty\right)$. Since $p t_{2} t^{p-1}+t^{p} \leq(p+1) t_{2} t^{p-1} \leq t_{2}^{p} \log ^{\lambda}\left(e+t_{2}\right)$ for any $t \ll 1$, we have that

$$
p t^{p-1}\left(t_{2}-t\right) \leq \Phi_{p, \lambda}\left(t_{2}\right)-t^{p}
$$

for all $t \ll 1$. Moreover when $t \leq t_{2}(p-1) /(p+1)$, we have that

$$
\frac{\Phi_{p, \lambda}\left(t_{2}\right)-t^{p}}{t_{2}-t} \leq \frac{\Phi_{p, \lambda}\left(t_{2}\right)}{t_{2}-t} \leq \frac{(p+1) \Phi_{p, \lambda}\left(t_{2}\right)}{2 t_{2}} .
$$

Therefore by (2.26), (2.27) and (2.28), there exists a constant $t_{1} \ll 1$ such that

$$
p t_{1}^{p-1} \leq \frac{\Phi_{p, \lambda}\left(t_{2}\right)-t_{1}^{p}}{t_{2}-t_{1}} \leq \frac{(p+1) \Phi_{p, \lambda}\left(t_{2}\right)}{2 t_{2}} \leq \Phi_{p, \lambda}^{\prime}\left(t_{2}\right) .
$$

Let $k=\left(\Phi_{p, \lambda}\left(t_{2}\right)-t_{1}^{p}\right) /\left(t_{2}-t_{1}\right)$. Given $p>1$ and $\lambda<0$, we define

$$
\Psi_{p, \lambda}(t)= \begin{cases}t^{p} & 0 \leq t<t_{1}, \\ k\left(t-t_{1}\right)+t_{1}^{p} & t_{1} \leq t<t_{2}, \\ \Phi_{p, \lambda}(t) & t_{2} \leq t .\end{cases}
$$


Proposition 2.9. The function $\Psi_{p, \lambda}$ is a Young function and satisfies the $\Delta_{2}$ condition on $[0, \infty)$. Moreover $i\left(\Psi_{p, \lambda}\right)=p$.

Proof. It is easy to see that $\Psi_{p, \lambda}$ is strictly increasing, continuous and convex on $[0,+\infty)$. Hence $\Psi_{p, \lambda}$ is a Young function. To prove the $\Delta_{2}$-condition, it suffices to check that

$$
\Psi_{p, \lambda}(2 t) \leq C \Psi_{p, \lambda}(t)
$$

for all $t \in[0,+\infty)$. In fact, $(2.30)$ is trivial if either $t \geq t_{2}$ or $2 t<t_{1}$. Whenever $t \in\left[t_{1} / 2, t_{2}\right]$, by the monotonicity of $\Psi_{p, \lambda}$ we have that

$$
\frac{\Psi_{p, \lambda}(2 t)}{\Psi_{p, \lambda}\left(2 t_{2}\right)} \leq 1 \leq \frac{\Psi_{p, \lambda}(t)}{\Psi_{p, \lambda}\left(t_{1} / 2\right)}
$$

Let $s \ll 1$. Without loss of generality, we assume $s \leq t_{1} / t_{2}$. In order to prove $i\left(\Psi_{p, \lambda}\right)=p$, we first estimate $h_{\Psi_{p, \lambda}}(s)$. By (2.29), we have that

$$
\frac{\Psi_{p, \lambda}(s t)}{\Psi_{p, \lambda}(t)}= \begin{cases}s^{p} & \forall t \in\left(0, t_{1}\right), \\ \frac{(s t)^{p}}{k\left(t-t_{1}\right)+t_{1}^{p}} \approx s^{p} & \forall t \in\left[t_{1}, t_{2}\right) .\end{cases}
$$

Moreover we obtain that

$$
\frac{s^{p}}{\log ^{\lambda}\left(e+t_{2}\right)} \leq \frac{\Psi_{p, \lambda}(s t)}{\Psi_{p, \lambda}(t)}=\frac{(s t)^{p}}{\Phi_{p, \lambda}(t)} \leq \frac{s^{p}}{\log ^{\lambda}\left(e+t_{1} s^{-1}\right)}
$$

for all $t \in\left[t_{2}, t_{1} / s\right)$ and

$$
\frac{t_{1}^{p} s^{p}}{t_{2}^{p} \log ^{\lambda}\left(e+t_{2} s^{-1}\right)} \leq \frac{\Psi_{p, \lambda}(s t)}{\Psi_{p, \lambda}(t)}=\frac{k\left(s t-t_{1}\right)+t_{1}^{p}}{\Phi_{p, \lambda}(t)} \leq \frac{\Phi_{p, \lambda}\left(t_{2}\right) s^{p}}{t_{1}^{p} \log ^{\lambda}\left(e+t_{1} s^{-1}\right)}
$$

for all $t \in\left[t_{1} / s, t_{2} / s\right)$. Assume $t \in\left[t_{2} / s,+\infty\right)$. It follows that

$$
\frac{\Psi_{p, \lambda}(s t)}{\Psi_{p, \lambda}(t)}=\frac{\Phi_{p, \lambda}(s t)}{\Phi_{p, \lambda}(t)}=s^{p}\left(\frac{\log (e+s t)}{\log (e+t)}\right)^{\lambda} .
$$

By the monotonicity of function $(\log (s)+\log (e+\cdot)) \log ^{-1}(e+\cdot)$, we have that

$$
\frac{\log (e+s t)}{\log (e+t)} \geq \frac{\log (s)+\log (e+t)}{\log (e+t)} \geq \frac{\log (s)+\log \left(e+t_{2} s^{-1}\right)}{\log \left(e+t_{2} s^{-1}\right)} \geq \frac{\log \left(t_{2}\right)}{\log \left(e+t_{2} s^{-1}\right)}
$$

for all $t \geq t_{2} / s$. Hence we derive from (2.34) that

$$
s^{p} \leq \frac{\Psi_{p, \lambda}(s t)}{\Psi_{p, \lambda}(t)} \leq \log ^{\lambda}\left(t_{2}\right) \frac{s^{p}}{\log ^{\lambda}\left(e+t_{2} s^{-1}\right)} .
$$


Combining (2.31), (2.32), (2.33) with (2.35) implies that

$$
s^{p} \lesssim h_{\Psi_{p, \lambda}}(s) \lesssim s^{p} \log ^{-\lambda}\left(s^{-1}\right)
$$

whenever $s \ll 1$. By (2.36), we therefore have that $i\left(\Psi_{p, \lambda}\right)=p$.

Remark 2.10. For $p>1$ and $\lambda<0$, let $\Psi_{p, \lambda}$ be as in (2.29) and $\Phi_{p, \lambda}$ be as in (2.21). Analogously to Remark 2.8, we have that

$$
\int_{\mathbb{R}^{2}} \Psi_{p, \lambda}\left(M_{f}(x)\right) w(x) d x \lesssim \int_{\mathbb{R}^{2}} \Psi_{p, \lambda}(|f(x)|) w(x) d x .
$$

Since $\lim _{t \rightarrow 0+} \Psi_{p, \lambda}(t) / \Phi_{p, \lambda}(t)=1$, it follows that

$$
\Psi_{p, \lambda}(t) \approx \Phi_{p, \lambda}(t)
$$

whenever $t \in[0,+\infty)$. Hence we derive from (2.37) that

$$
\int_{\mathbb{R}^{2}} \Phi_{p, \lambda}\left(M_{f}(x)\right) w(x) d x \lesssim \int_{\mathbb{R}^{2}} \Phi_{p, \lambda}(|f(x)|) w(x) d x .
$$

\section{Proof of Theorem 1.1}

We begin by proving the following special case of Theorem 1.1.

Theorem 3.1. Let $\varphi: \mathbb{S}^{1} \rightarrow \mathbb{S}^{1}$ be a homeomorphism, and $h=P[\varphi]: \mathbb{D} \rightarrow \mathbb{D}$ be the harmonic extension of $\varphi$. For any $p>1$, we have that

(1) if either $\alpha \in(p-2,+\infty)$ and $\lambda \in \mathbb{R}$, or $\alpha=p-2$ and $\lambda \in(-\infty,-1)$,

$$
\text { both } I_{1}(p, \alpha, \lambda, h) \text { and } I_{2}(p, \alpha, \lambda, h) \text { are finite. }
$$

(2) if either $\alpha \in(-1, p-2)$ and $\lambda \in \mathbb{R}$, or $\alpha=p-2$ and $\lambda \in[-1,+\infty)$, then both $I_{1}(p, \alpha, \lambda, h)$ and $I_{2}(p, \alpha, \lambda, h)$ are comparable to $\mathcal{U}(p, \alpha, \lambda, \varphi)$.

Moreover whenever $p \in(1,2]$

both $I_{1}(p, \alpha, \lambda, h)$ and $I_{2}(p, \alpha, \lambda, h)$ dominate $\mathcal{V}(p, \alpha, \lambda, \varphi)$,

while

$$
\mathcal{V}(p, \alpha, \lambda, \varphi) \text { controls both } I_{1}(p, \alpha, \lambda, h) \text { and } I_{2}(p, \alpha, \lambda, h)
$$

for all $p \in[2,+\infty)$. Furthermore both $I_{1}(p, \alpha, \lambda, h)$ and $I_{2}(p, \alpha, \lambda, h)$ are in general comparable to $\mathcal{V}(p, \alpha, \lambda, \varphi)$ only for $p=2$. 
(3) if either $\alpha \in(-\infty,-1)$ and $\lambda \in \mathbb{R}$, or $\alpha=-1$ and $\lambda \in[-1,+\infty)$, we have that $I_{1}(p, \alpha, \lambda, h)=\infty$. While $I_{2}(p, \alpha, \lambda, h)=\infty$ for all $\alpha \in(-\infty,-1]$ and $\lambda \in \mathbb{R}$.

Let $\varphi: \mathbb{S}^{1} \rightarrow \mathbb{S}^{1}$ be a homeomorphism. Given $p>1, \alpha \in \mathbb{R}, \lambda \in \mathbb{R}$, we define

$$
\mathcal{E}_{1}(p, \alpha, \lambda, \varphi)=\sum_{j=1}^{+\infty} \sum_{k=1}^{2^{j}} \ell\left(\varphi\left(\Gamma_{j, k}\right)\right)^{p} \ell\left(\Gamma_{j, k}\right)^{2+\alpha-p} j^{\lambda}
$$

and

$$
\mathcal{E}_{2}(p, \alpha, \lambda, \varphi)=\sum_{j=1}^{+\infty} \sum_{k=1}^{2^{j}} \Phi_{p, \lambda}\left(\frac{\ell\left(\varphi\left(\Gamma_{j, k}\right)\right)}{\ell\left(\Gamma_{j, k}\right)}\right) \ell\left(\Gamma_{j, k}\right)^{2+\alpha}
$$

where $\Phi_{p, \lambda}(t)$ is from $(2.21)$.

Lemma 3.2. Let $\varphi: \mathbb{S}^{1} \rightarrow \mathbb{S}^{1}$ be a homeomorphism. For any $p>1, \alpha \in$ $(-1,+\infty)$ and every $\lambda \in \mathbb{R}$, the dyadic energies $\mathcal{E}_{1}(p, \alpha, \lambda, \varphi)$ and $\mathcal{E}_{2}(p, \alpha, \lambda, \varphi)$ are equivalent.

Proof. We first consider the case $\lambda \geq 0$. Let $\Phi_{p, \lambda}$ be as in (2.21). Since $\ell\left(\varphi\left(\Gamma_{j, k}\right)\right) \leq$ $2 \pi$ and $\ell\left(\Gamma_{j, k}\right) \approx 2^{-j}$ for all $j \in \mathbb{N}$ and $k \in\left\{1, \ldots, 2^{j}\right\}$, by the monotonicity and $\Delta_{2}$-property of the standard logarithm we have that

$$
\Phi_{p, \lambda}\left(\frac{\ell\left(\varphi\left(\Gamma_{j, k}\right)\right)}{\ell\left(\Gamma_{j, k}\right)}\right) \lesssim\left(\frac{\ell\left(\varphi\left(\Gamma_{j, k}\right)\right)}{\ell\left(\Gamma_{j, k}\right)}\right)^{p} \log ^{\lambda}\left(e+2 \pi \cdot 2^{j}\right) \lesssim\left(\frac{\ell\left(\varphi\left(\Gamma_{j, k}\right)\right)}{\ell\left(\Gamma_{j, k}\right)}\right)^{p} j^{\lambda} .
$$

Hence

$$
\mathcal{E}_{2}(p, \alpha, \lambda, \varphi) \lesssim \mathcal{E}_{1}(p, \alpha, \lambda, \varphi)
$$

Given $p>1$ and $\alpha \in(-1,+\infty)$, there is $\beta \in(0,1)$ such that $\alpha>(1-\beta) p-1>$ -1 . Define

$$
\chi_{j, k}= \begin{cases}1 & \text { if } \ell\left(\varphi\left(\Gamma_{j, k}\right)\right) \geq 2^{-j \beta} \\ 0 & \text { otherwise }\end{cases}
$$

We decompose $\mathcal{E}_{1}(p, \alpha, \lambda, \varphi)$ as

$$
\begin{aligned}
\mathcal{E}_{1}(p, \alpha, \lambda, \varphi)= & \sum_{j=1}^{+\infty} \sum_{k=1}^{2^{j}} \ell\left(\varphi\left(\Gamma_{j, k}\right)\right)^{p} \ell\left(\Gamma_{j, k}\right)^{2+\alpha-p} j^{\lambda} \chi_{j, k} \\
& +\sum_{j=1}^{+\infty} \sum_{k=1}^{2^{j}} \ell\left(\varphi\left(\Gamma_{j, k}\right)\right)^{p} \ell\left(\Gamma_{j, k}\right)^{2+\alpha-p} j^{\lambda}\left(1-\chi_{j, k}\right) \\
= & : \mathcal{E}_{1}^{\prime}(p, \alpha, \lambda, \varphi)+\mathcal{E}_{1}^{\prime \prime}(p, \alpha, \lambda, \varphi) .
\end{aligned}
$$


Whenever $\ell\left(\varphi\left(\Gamma_{j, k}\right)\right) \geq 2^{-j \beta}$, by $(2.3)$ we have $j^{\lambda} \lesssim \log ^{\lambda}\left(e+\ell\left(\varphi\left(\Gamma_{j, k}\right)\right) \ell\left(\Gamma_{j, k}\right)^{-1}\right)$. Therefore

$$
\begin{aligned}
\mathcal{E}_{1}^{\prime}(p, \alpha, \lambda, \varphi) & \lesssim \sum_{j=1}^{+\infty} \sum_{k=1}^{2^{j}} \ell\left(\varphi\left(\Gamma_{j, k}\right)\right)^{p} \ell\left(\Gamma_{j, k}\right)^{2+\alpha-p} \log ^{\lambda}\left(e+\frac{\ell\left(\varphi\left(\Gamma_{j, k}\right)\right)}{\ell\left(\Gamma_{j, k}\right)}\right) \\
& =\mathcal{E}_{2}(p, \alpha, \lambda, \varphi) .
\end{aligned}
$$

Moreover, by (2.3) we have that

$$
\mathcal{E}_{1}^{\prime \prime}(p, \alpha, \lambda, \varphi) \leq \sum_{j=1}^{+\infty} \sum_{k=1}^{2^{j}} 2^{-\beta j p} 2^{j(p-2-\alpha)} j^{\lambda}=\sum_{j=1}^{+\infty} 2^{-j((\beta-1) p+1+\alpha)} j^{\lambda}<\infty .
$$

We conclude from (3.2), (3.3) and (3.4) that there is a constant $C>0$ such that

$$
\mathcal{E}_{1}(p, \alpha, \lambda, \varphi) \lesssim C+\mathcal{E}_{2}(p, \alpha, \lambda, \varphi) .
$$

From (3.1) and (3.5) it follows that

$$
\mathcal{E}_{1}(p, \alpha, \lambda, \varphi) \text { and } \mathcal{E}_{2}(p, \alpha, \lambda, \varphi) \text { are comparable whenever } \lambda \geq 0 .
$$

Analogously to (3.6), we have that $\mathcal{E}_{1}(p, \alpha, \lambda, \varphi)$ and $\mathcal{E}_{2}(p, \alpha, \lambda, \varphi)$ are comparable whenever $\lambda<0$.

Lemma 3.3. Let $\varphi: \mathbb{S}^{1} \rightarrow \mathbb{S}^{1}$ be a homeomorphism, and $h=P[\varphi]: \mathbb{D} \rightarrow \mathbb{D}$ be the Poisson homeomorphic extension of $\varphi$. For any $p>1$, we have that $I_{1}(p, \alpha, \lambda, h) \lesssim \mathcal{E}_{1}(p, \alpha, \lambda, \varphi)$ whenever $\alpha \in(-1,+\infty)$ and $\lambda \in \mathbb{R}$, while $\mathcal{E}_{1}(p, \alpha, \lambda, \varphi)$ is controlled by $I_{1}(p, \alpha, \lambda, h)$ for all $\alpha \in(-1, p-1)$ and $\lambda \in \mathbb{R}$.

Proof. We first prove that $I_{1}(p, \alpha, \lambda, h) \lesssim \mathcal{E}_{1}(p, \alpha, \lambda, \varphi)$ for all $\alpha>-1$ and all $\lambda \in \mathbb{R}$. Let $w_{\alpha, \lambda}$ be as in (2.7). For any $j \in \mathbb{N}$ and $1 \leq k \leq 2^{j}$, by (2.4) and (2.3) we have that

$$
w_{\alpha, \lambda}(z) \approx 2^{-j \alpha} j^{\lambda} \approx \ell\left(\Gamma_{j, k}\right)^{\alpha} j^{\lambda}
$$

for all $z \in Q_{j, k}$. Hence

$$
I_{1}(p, \alpha, \lambda, h) \approx \sum_{j=1}^{+\infty} \sum_{k=1}^{2^{j}} 2^{-j \alpha} j^{\lambda} \int_{Q_{j, k}}|D h(z)|^{p} d z .
$$

Let $\mathcal{P}\left(\Gamma_{j, k}\right)$ be the technical decomposition of $\mathbb{S}^{1}$ based on $\Gamma_{j, k}$ in [9, Section 2.1]. As shown in $[9$, Proof (iv) $\Rightarrow(\mathrm{i})]$, for any $j \in \mathbb{N}$ and $k=1, \ldots, 2^{j}$ we have that

$$
|D h(z)| \lesssim \sum_{n \leq j} \sum_{m \in i_{n}} \frac{\ell\left(\varphi\left(\Gamma_{n, m}\right)\right)}{2^{-n}}
$$


for all $z \in Q_{j, k}$. Here $\Gamma_{n, m} \in \mathcal{P}\left(\Gamma_{j, k}\right)$ and $\sharp i_{n} \leq 3$ for all $n \leq j$, see $[9$, Section 2.1]. Let $\alpha>-1$. There is $q_{0}>1$ such that $p / q_{0}-1-\alpha<0$. Denote by $p_{0}$ the exponent conjugate to $q_{0}$. Via Hölder's inequality we derive from (3.9) that

$$
\begin{aligned}
|D h(z)|^{p} & \lesssim\left(\sum_{n \leq j} \sum_{m \in i_{n}} \frac{\ell\left(\varphi\left(\Gamma_{n, m}\right)\right)}{2^{-n\left(\frac{1}{q_{0}}+\frac{1}{p_{0}}\right)}}\right)^{p} \leq\left(\sum_{n \leq j} \sum_{m \in i_{n}} 2^{\frac{n q}{q_{0}}}\right)^{\frac{p}{q}} \sum_{n \leq j} \sum_{m \in i_{n}} \frac{\ell\left(\varphi\left(\Gamma_{n, m}\right)\right)^{p}}{2^{-\frac{n p}{p_{0}}}} \\
& \approx 2^{\frac{j p}{q_{0}}} \sum_{n \leq j} \sum_{m \in i_{n}} \frac{\ell\left(\varphi\left(\Gamma_{n, m}\right)\right)^{p}}{2^{-\frac{n p}{p_{0}}}}
\end{aligned}
$$

for all $z \in Q_{j, k}$. By (2.5), (3.8) and (3.10) we have that

$$
I_{1}(p, \alpha, \lambda, h) \lesssim \sum_{j=1}^{+\infty} \sum_{k=1}^{2^{j}} 2^{j\left(\frac{p}{q_{0}}-2-\alpha\right)} j^{\lambda} \sum_{n \leq j} \sum_{m \in i_{n}} \frac{\ell\left(\varphi\left(\Gamma_{n, m}\right)\right)^{p}}{2^{-\frac{n p}{q_{0}}}} .
$$

Moreover given a dyadic arc $\Gamma_{n, m}$, for any $j \geq n$ it is shown in $[9$, Section 2.1] that

$$
\sharp\left\{\Gamma: \Gamma \text { is a } j \text {-level dyadic arc and } \Gamma_{n, m} \in \mathcal{P}(\Gamma)\right\} \leq 3 \cdot 2^{j-n} \text {. }
$$

From Fubini's theorem and (3.12) we obtain that

$$
\begin{aligned}
& \sum_{j=1}^{+\infty} \sum_{k=1}^{2^{j}} 2^{j\left(\frac{p}{q_{0}}-2-\alpha\right)} j^{\lambda} \sum_{n \leq j} \sum_{m \in i_{n}} \frac{\ell\left(\varphi\left(\Gamma_{n, m}\right)\right)^{p}}{2^{-\frac{n p}{p_{0}}}} \\
= & \sum_{n=1}^{\infty} \sum_{m=1}^{2^{n}} \frac{\ell\left(\varphi\left(\Gamma_{n, m}\right)\right)^{p}}{2^{-\frac{n p}{p_{0}}}} \sum_{n \leq j} \sum_{k} 2^{j\left(\frac{p}{q_{0}}-2-\alpha\right)} j^{\lambda} \\
\lesssim & \sum_{n=1}^{\infty} \sum_{m=1}^{2^{n}} \frac{\ell\left(\varphi\left(\Gamma_{n, m}\right)\right)^{p}}{2^{-\frac{n p}{p_{0}}}} \sum_{n \leq j} 2^{j\left(\frac{p}{q_{0}}-2-\alpha\right)} j^{\lambda} 2^{j-n} \\
= & \sum_{n=1}^{\infty} \sum_{m=1}^{2^{n}} \ell\left(\varphi\left(\Gamma_{n, m}\right)\right)^{p} 2^{n\left(\frac{p}{p_{0}}-1\right)} \sum_{n \leq j} 2^{j\left(\frac{p}{q_{0}}-1-\alpha\right)} j^{\lambda} .
\end{aligned}
$$

Moreover when $p / q_{0}-1-\alpha<0$ we have that

$$
\sum_{n \leq j} 2^{j\left(\frac{p}{q_{0}}-1-\alpha\right)} j^{\lambda} \approx 2^{n\left(\frac{p}{q_{0}}-1-\alpha\right)} n^{\lambda} .
$$

By (3.11), (3.13), (3.14) and (2.3), we conclude that

$$
I_{1}(p, \alpha, \lambda, h) \lesssim \sum_{n=1}^{\infty} \sum_{m=1}^{2^{n}} \ell\left(\varphi\left(\Gamma_{n, m}\right)\right)^{p} 2^{n(p-2-\alpha)} n^{\lambda} \approx \mathcal{E}_{1}(p, \alpha, \lambda, \varphi) .
$$


We next prove that $\mathcal{E}_{1}(p, \alpha, \lambda, \varphi)$ is controlled by $I_{1}(p, \alpha, \lambda, h)$ for all $\alpha \in$ $(-1, p-1)$ and $\lambda \in \mathbb{R}$. By $[9,(3.17)]$, there is $j_{0}>1$ such that

$$
\ell\left(\varphi\left(\Gamma_{j, k}\right)\right) \lesssim \frac{1}{\ell\left(\Gamma_{j, k}\right)} \int_{C Q_{j, k} \cap \mathbb{D}}|D h(z)| d z
$$

for all $j \geq j_{0}$ and $k \in\left\{1, \ldots, 2^{j}\right\}$. Set $H(z)=|D h(z)| \chi_{\mathbb{D}}(z)$. By (2.6) we have that

$$
f_{C Q_{j, k} \cap \mathbb{D}}|D h(z)| d z \leq f_{C C^{\prime} B_{j, k}} H(z) d z \leq f_{Q_{j, k}} M_{H}(w) d w
$$

where the last inequality comes from the fact that $f_{C C^{\prime} B_{j, k}} H(z) d z \leq M_{H}(w)$ for all $w \in Q_{j, k}$. Combining (3.15) with (3.16) implies that

$$
\ell\left(\varphi\left(\Gamma_{j, k}\right)\right) \lesssim \ell\left(\Gamma_{j, k}\right) f_{Q_{j, k}} M_{H}(z) d z
$$

for all $j \geq j_{0}$ and $k=1, \ldots, 2^{j}$. By Jensen's inequality and (2.5), we deduce from (3.17) that

$$
\ell\left(\varphi\left(\Gamma_{j, k}\right)\right)^{p} \lesssim \ell\left(\Gamma_{j, k}\right)^{p-2} \int_{Q_{j, k}} M_{H}^{p}(z) d z
$$

By (3.7) and (3.18), there is then a constant $C>0$ such that

$$
\mathcal{E}_{1}(p, \alpha, \lambda, \varphi) \lesssim C+\sum_{j=j_{0}}^{+\infty} \sum_{k=1}^{2^{j}} \int_{Q_{j, k}} M_{H}^{p}(z) w_{\alpha, \lambda}(z) d z \leq C+\int_{\mathbb{R}^{2}} M_{H}^{p}(z) w_{\alpha, \lambda}(z) d z .
$$

Moreover, for any $\alpha \in(-1, p-1)$ and $\lambda \in \mathbb{R}$, from Proposition 2.3 and Remark 2.8 it follows that

$$
\int_{\mathbb{R}^{2}} M_{H}^{p}(z) w_{\alpha, \lambda}(z) d z \lesssim \int_{\mathbb{R}^{2}} H^{p}(z) w_{\alpha, \lambda}(z) d z=I_{1}(p, \alpha, \lambda, h) .
$$

By (3.19) and (3.20) we conclude that $\mathcal{E}_{1}(p, \alpha, \lambda, \varphi)$ is controlled by $I_{1}(p, \alpha, \lambda, h)$ for all $\alpha \in(-1, p-1)$ and $\lambda \in \mathbb{R}$.

Lemma 3.4. Let $\varphi: \mathbb{S}^{1} \rightarrow \mathbb{S}^{1}$ be a homeomorphism, and $h=P[\varphi]: \mathbb{D} \rightarrow \mathbb{D}$ be the Poisson homeomorphic extension of $\varphi$. For any $p>1$, we have that $I_{2}(p, \alpha, \lambda, h) \lesssim \mathcal{E}_{1}(p, \alpha, \lambda, \varphi)$ whenever $\alpha \in(-1,+\infty)$ and $\lambda \in \mathbb{R}$, while $\mathcal{E}_{2}(p, \alpha, \lambda, \varphi)$ is controlled by $I_{2}(p, \alpha, \lambda, h)$ for all $\alpha \in(-1, p-1)$ and $\lambda \in \mathbb{R}$. 
Proof. We first consider that case $\lambda \geq 0$. Let $\Phi_{p, \lambda}$ be as in (2.21). Proposition 2.7 shows that $\Phi_{p, \lambda}(t)$ is increasing and satisfies $\Delta_{2}$-property on $[0,+\infty)$. From (3.7) and (3.9) we have that

$$
\begin{aligned}
I_{2}(p, \alpha, \lambda, h) & =\sum_{j=1}^{+\infty} \sum_{k=1}^{2^{j}} \int_{Q_{j, k}} \Phi_{p, \lambda}(|D h(z)|) w_{\alpha, 0}(z) d z \\
& \lesssim \sum_{j=1}^{+\infty} \sum_{k=1}^{2^{j}} \ell\left(\Gamma_{j, k}\right)^{\alpha+2} \Phi_{p, \lambda}\left(\sum_{n \leq j} \sum_{m \in i_{n}} \frac{\ell\left(\varphi\left(\Gamma_{n, m}\right)\right)}{2^{-n}}\right) .
\end{aligned}
$$

Moreover since $\ell\left(\varphi\left(\Gamma_{n, m}\right)\right) \leq 2 \pi$ for all $n \in \mathbb{N}$ and $m=1, \ldots, 2^{n}$, it follows that

$$
\sum_{n \leq j} \sum_{m \in i_{n}} \frac{\ell\left(\varphi\left(\Gamma_{n, m}\right)\right)}{2^{-n}} \lesssim \sum_{n \leq j} \frac{1}{2^{-n}} \lesssim 2^{j}
$$

for any $j \geq 1$. Therefore

$$
\log ^{\lambda}\left(e+\sum_{n \leq j} \sum_{m} \frac{\ell\left(\varphi\left(\Gamma_{n, m}\right)\right)}{2^{-n}}\right) \lesssim \log ^{\lambda}\left(e+2^{j}\right) \lesssim j^{\lambda}
$$

for all $j \geq 1$. By (3.21) and (3.22) we obtain that

$$
I_{2}(p, \alpha, \lambda, h) \lesssim \sum_{j=1}^{+\infty} \sum_{k=1}^{2^{j}} \ell\left(\Gamma_{j, k}\right)^{\alpha+2} j^{\lambda}\left(\sum_{n \leq j} \sum_{m \in i_{n}} \frac{\ell\left(\varphi\left(\Gamma_{n, m}\right)\right)}{2^{-n}}\right)^{p} .
$$

The analogous arguments as for $I_{1}(p, \alpha, \lambda, h) \lesssim \mathcal{E}_{1}(p, \alpha, \lambda, \varphi)$ in Lemma 3.3 imply that

$$
\sum_{j=1}^{+\infty} \sum_{k=1}^{2^{j}} \ell\left(\Gamma_{j, k}\right)^{\alpha+2} j^{\lambda}\left(\sum_{n \leq j} \sum_{m \in i_{n}} \frac{\ell\left(\varphi\left(\Gamma_{n, m}\right)\right)}{2^{-n}}\right)^{p} \lesssim \mathcal{E}_{1}(p, \alpha, \lambda, \varphi) .
$$

We conclude from (3.23) and (3.24) that $I_{2}(p, \alpha, \lambda, h) \lesssim \mathcal{E}_{1}(p, \alpha, \lambda, \varphi)$.

Applying $\Phi_{p, \lambda}$ to the both sides of (3.17), via Proposition 2.7 and Jensen's inequality we have that

$$
\Phi_{p, \lambda}\left(\frac{\ell\left(\varphi\left(\Gamma_{j, k}\right)\right)}{\ell\left(\Gamma_{j, k}\right)}\right) \lesssim \Phi_{p, \lambda}\left(f_{Q_{j, k}} M_{H}(z) d z\right) \leq f_{Q_{j, k}} \Phi_{p, \lambda}\left(M_{H}(z)\right) d z
$$

for all $j \geq j_{0}$ and $k \in\left\{1, \ldots, 2^{j}\right\}$. By (2.5), (3.7) and (3.25), we then obtain that

$$
\begin{aligned}
\mathcal{E}_{2}(p, \alpha, \lambda, \varphi) & \lesssim \sum_{j=1}^{+\infty} \sum_{k=1}^{2^{j}} \int_{Q_{j, k}} \Phi_{p, \lambda}\left(M_{H}(z)\right) w_{\alpha, 0}(z) d z \\
& \leq \int_{\mathbb{R}^{2}} \Phi_{p, \lambda}\left(M_{H}(z)\right) w_{\alpha, 0}(z) d z
\end{aligned}
$$


Moreover, for any $\alpha \in(-1, p-1)$ it follows from Remark 2.8 that

$$
\int_{\mathbb{R}^{2}} \Phi_{p, \lambda}\left(M_{H}(z)\right) w_{\alpha, 0}(z) d z \lesssim \int_{\mathbb{R}^{2}} \Phi_{p, \lambda}(H(z)) w_{\alpha, 0}(z) d z=I_{2}(p, \alpha, \lambda, h) .
$$

By (3.26) and (3.27) we conclude that $\mathcal{E}_{2}(p, \alpha, \lambda, \varphi) \lesssim I_{2}(p, \alpha, \lambda, h)$.

We next consider the case $\lambda<0$. Let $\Psi_{p, \lambda}$ be as in (2.29). By the analogous arguments as for (3.21), we have that

$$
\int_{\mathbb{D}} \Psi_{p, \lambda}(|D h(z)|) w_{\alpha, 0}(z) d z \lesssim \sum_{j=1}^{+\infty} \sum_{k=1}^{2^{j}} \ell\left(\Gamma_{j, k}\right)^{\alpha+2} \Psi_{p, \lambda}\left(\sum_{n \leq j} \sum_{m \in i_{n}} \frac{\ell\left(\varphi\left(\Gamma_{n, m}\right)\right)}{2^{-n}}\right) .
$$

Set $S_{j, k}=\sum_{n \leq j} \sum_{m \in i_{n}} \frac{\ell\left(\varphi\left(\Gamma_{n, m}\right)\right)}{2^{-n}}$. It follows from (2.38) and (3.28) that

$$
I_{2}(p, \alpha, \lambda, h) \lesssim \sum_{j=1}^{+\infty} \sum_{k=1}^{2^{j}} \ell\left(\Gamma_{j, k}\right)^{\alpha+2} \Phi_{p, \lambda}\left(S_{j, k}\right) .
$$

Since $\alpha>-1$, there is $\beta>0$ such that $\beta p \leq 1+\alpha$. Define

$$
\chi(j, k)= \begin{cases}1 & \text { if } S_{j, k}<2^{j \beta} \\ 0 & \text { otherwise. }\end{cases}
$$

Then

$$
\begin{aligned}
\sum_{j=1}^{+\infty} \sum_{k=1}^{2^{j}} \ell\left(\Gamma_{j, k}\right)^{\alpha+2} \Phi_{p, \lambda}\left(S_{j, k}\right)= & \sum_{j=1}^{+\infty} \sum_{k=1}^{2^{j}} \ell\left(\Gamma_{j, k}\right)^{\alpha+2} \Phi_{p, \lambda}\left(\chi(j, k) S_{j, k}\right) \\
& +\sum_{j=1}^{+\infty} \sum_{k=1}^{2^{j}} \ell\left(\Gamma_{j, k}\right)^{\alpha+2} \Phi_{p, \lambda}\left((1-\chi(j, k)) S_{j, k}\right)=: \sum_{1}+\sum_{2} .
\end{aligned}
$$

Since $\log ^{\lambda}\left(e+S_{j, k}\right) \leq \log ^{\lambda}(e)=1$, we have that

$$
\sum_{1} \leq \sum_{1} 2^{-j(\alpha+2)}\left(S_{j, k}\right)^{p} \leq \sum_{j=1}^{\infty} 2^{j(p \beta-\alpha-1)}<\infty
$$

Whenever $S_{j, k} \geq 2^{j \beta}$, it follows that $\log ^{\lambda}\left(e+S_{j, k}\right) \lesssim j^{\lambda}$. Via the analogous arguments as for $I_{1}(p, \alpha, \lambda, h) \lesssim \mathcal{E}_{1}(p, \alpha, \lambda, \varphi)$ in Lemma 3.3, it then follows that

$$
\sum_{2} \leq \sum_{j=1}^{\infty} \sum_{k=1}^{2^{j}} \ell\left(\Gamma_{j, k}\right)^{\alpha+2} j^{\lambda} S_{j, k}^{p} \lesssim \mathcal{E}_{1}(p, \alpha, \lambda, \varphi)
$$


From $(3.29),(3.30),(3.31)$ and (3.32), we conclude that there is a constant $C>0$ such that $I_{2}(p, \alpha, \lambda, h) \lesssim C+\mathcal{E}_{1}(p, \alpha, \lambda, \varphi)$.

By the analogous arguments as for $\mathcal{E}_{2}(p, \alpha, \lambda, \varphi) \lesssim I_{2}(p, \alpha, \lambda, h)$ whenever $\lambda \geq 0$, we have that

$$
\sum_{j=1}^{+\infty} \sum_{k=1}^{2^{j}} \ell\left(\Gamma_{j, k}\right)^{\alpha+2} \Psi_{p, \lambda}\left(\frac{\ell\left(\varphi\left(\Gamma_{j, k}\right)\right)}{\ell\left(\Gamma_{j, k}\right)}\right) \lesssim \int_{\mathbb{R}^{2}} \Psi_{p, \lambda}(|D h|(z)) w_{\alpha, 0}(z) d z
$$

It follows from $(2.38)$ that $\mathcal{E}_{2}(p, \alpha, \lambda, \varphi) \lesssim I_{2}(p, \alpha, \lambda, h)$.

Proof of Theorem 3.1 (1). From Lemma 3.3 and Lemma 3.4, we have that both $I_{1}(p, \alpha, \lambda, h)$ and $I_{2}(p, \alpha, \lambda, h)$ are dominated by $\mathcal{E}_{1}(p, \alpha, \lambda, \varphi)$ for all $p>$ $1, \alpha \in(-1,+\infty)$ and each $\lambda \in \mathbb{R}$. Moreover since $\ell\left(\varphi\left(\Gamma_{j, k}\right)\right) \leq 2 \pi$ for all $j \geq 1$ and $1 \leq k \leq 2^{j}$, we have that

$$
\sum_{k=1}^{2^{j}} \ell\left(\varphi\left(\Gamma_{j, k}\right)\right)^{p} \leq(2 \pi)^{p-1} \sum_{k=1}^{2^{j}} \ell\left(\varphi\left(\Gamma_{j, k}\right)\right)=(2 \pi)^{p}
$$

Therefore both $I_{1}(p, \alpha, \lambda, h)$ and $I_{2}(p, \alpha, \lambda, h)$ are controlled by $\sum_{j=1}^{\infty} 2^{j(p-2-\alpha)} j^{\lambda}$ whenever $\alpha \in(-1,+\infty)$ and $\lambda \in \mathbb{R}$. Notice that $\sum_{j=1}^{\infty} 2^{j(p-2-\alpha)} j^{\lambda}<\infty$ whenever either $p-2<\alpha$ and $\lambda \in \mathbb{R}$, or $p-2=\alpha$ and $\lambda<-1$. We hence complete Theorem 3.1 (1).

By Example 4.4, there are homeomorphisms $\varphi: \mathbb{S}^{1} \rightarrow \mathbb{S}^{1}$ such that, for their harmonic extensions $P[\varphi]$, both $I_{1}(p, \alpha, \lambda, P[\varphi])$ and $I_{2}(p, \alpha, \lambda, P[\varphi])$ may be finite or infinite for either some $\alpha \in(-1, p-2)$ and $\lambda \in \mathbb{R}$ or some $\alpha=p-2$ and $\lambda \in[-1,+\infty)$. How can we characterize both $I_{1}(p, \alpha, \lambda, P[\varphi])<\infty$ and $I_{2}(p, \alpha, \lambda, P[\varphi])<\infty$ ? As shown in [9], double integrals of the inverse mapping over the boundary are potential choices.

Lemma 3.5. Let $\varphi: \mathbb{S}^{1} \rightarrow \mathbb{S}^{1}$ be a homeomorphism. For any $\alpha \in \mathbb{R}$ and $\lambda \in \mathbb{R}$, $\mathcal{V}(p, \alpha, \lambda, \varphi)$ is dominated by $\mathcal{E}_{1}(p, \alpha, \lambda, \varphi)$ whenever $p \in(1,2]$; while $\mathcal{E}_{1}(p, \alpha, \lambda, \varphi)$ is controlled by $\mathcal{V}(p, \alpha, \lambda, \varphi)$ if $p \in[2,+\infty)$.

Proof. We first consider the case $p \in(1,2]$. Given $\xi \in \mathbb{S}^{1}$ and $t \geq 0$, set

$$
E_{t}(\xi)=\left\{\eta \in \mathbb{S}^{1}:\left|\varphi^{-1}(\xi)-\varphi^{-1}(\eta)\right|<t\right\} .
$$


By Fubini's theorem we have that

$$
\begin{aligned}
\int_{\mathbb{S}^{1}} \mathcal{A}_{p, \alpha, \lambda}\left(\left|\varphi^{-1}(\xi)-\varphi^{-1}(\eta)\right|\right)|d \eta| & =\int_{\mathbb{S}^{1}} \int_{\left|\varphi^{-1}(\xi)-\varphi^{-1}(\eta)\right|}^{1}-\mathcal{A}_{p, \alpha, \lambda}^{\prime}(t) d t|d \eta| \\
& =\int_{0}^{1} \int_{\mathbb{S}^{1}}-\mathcal{A}_{p, \alpha, \lambda}^{\prime}(t) \chi_{E_{t}(\xi)}|d \eta| d t \\
& =\int_{0}^{1}-\mathcal{A}_{p, \alpha, \lambda}^{\prime}(t) \mathcal{L}^{1}\left(E_{t}(\xi)\right) d t
\end{aligned}
$$

Moreover, from Jensen's inequality and Minkowski's inequality it follows that

$$
\begin{aligned}
& \left(\int_{\mathbb{S}^{1}}\left(\int_{0}^{1}-\mathcal{A}_{p, \alpha, \lambda}^{\prime}(t) \mathcal{L}^{1}\left(E_{t}(\xi)\right) d t\right)^{p-1}|d \xi|\right)^{\frac{1}{p-1}} \\
\lesssim & \left(\int_{\mathbb{S}^{1}}\left(\int_{0}^{1}-\mathcal{A}_{p, \alpha, \lambda}^{\prime}(t) \mathcal{L}^{1}\left(E_{t}(\xi)\right) d t\right)^{\frac{1}{p-1}}|d \xi|\right)^{p-1} \\
\leq & \int_{0}^{1}\left(\int_{\mathbb{S}^{1}}\left(-\mathcal{A}_{p, \alpha, \lambda}^{\prime}(t) \mathcal{L}^{1}\left(E_{t}(\xi)\right)\right)^{\frac{1}{p-1}}|d \xi|\right)^{p-1} d t \\
= & \int_{0}^{1}-\mathcal{A}_{p, \alpha, \lambda}^{\prime}(t)\left(\int_{\mathbb{S}^{1}} \mathcal{L}^{1}\left(E_{t}(\xi)\right)^{\frac{1}{p-1}}|d \xi|\right)^{p-1} d t .
\end{aligned}
$$

Combining (3.34) with (3.35) implies that

$$
\begin{aligned}
\mathcal{V}^{\frac{1}{p-1}}(p, \alpha, \lambda, \varphi) & \lesssim \int_{0}^{1}-\mathcal{A}_{p, \alpha, \lambda}^{\prime}(t)\left(\int_{\mathbb{S}^{1}} \mathcal{L}^{1}\left(E_{t}(\xi)\right)^{\frac{1}{p-1}}|d \xi|\right)^{p-1} d t \\
& \leq \sum_{j=1}^{+\infty} \int_{2^{-j}}^{2^{1-j}}-\mathcal{A}_{p, \alpha, \lambda}^{\prime}(t) d t\left(\int_{\mathbb{S}^{1}} \mathcal{L}^{1}\left(E_{2^{1-j}}(\xi)\right)^{\frac{1}{p-1}}|d \xi|\right)^{p-1}
\end{aligned}
$$

Since $E_{2^{1-j}}(\xi) \subset \cup_{i=k-1}^{k+1} \varphi\left(\Gamma_{j, i}\right)$ for all $j \in \mathbb{N}, k=1, \ldots, 2^{j}$ and all $\xi \in \varphi\left(\Gamma_{j, k}\right)$, we have that

$$
\begin{aligned}
\left(\int_{\mathbb{S}^{1}} \mathcal{L}^{1}\left(E_{2^{1-j}}(\xi)\right)^{\frac{1}{p-1}}|d \xi|\right)^{p-1} & =\left(\sum_{k=1}^{2^{j}} \int_{\varphi\left(\Gamma_{j, k}\right)} \mathcal{L}^{1}\left(E_{2^{1-j}}(\xi)\right)^{\frac{1}{p-1}}|d \xi|\right)^{p-1} \\
& \leq\left(\sum_{k=1}^{2^{j}} \ell\left(\varphi\left(\Gamma_{j, k}\right)\right)\left(\sum_{i=k-1}^{k+1} \ell\left(\varphi\left(\Gamma_{j, i}\right)\right)\right)^{\frac{1}{p-1}}\right)^{p-1} \\
& \leq \sum_{k=1}^{2^{j}} \ell\left(\varphi\left(\Gamma_{j, k}\right)\right)^{p-1} \sum_{i=k-1}^{k+1} \ell\left(\varphi\left(\Gamma_{j, i}\right)\right) .
\end{aligned}
$$


Moreover by Young's inequality we have that

$$
\begin{aligned}
\sum_{k=1}^{2^{j}} \ell\left(\varphi\left(\Gamma_{j, k}\right)\right)^{p-1} \ell\left(\varphi\left(\Gamma_{j, k-1}\right)\right) & \leq \sum_{k=1}^{2^{j}} \frac{1}{p} \ell\left(\varphi\left(\Gamma_{j, k}\right)\right)^{p}+\frac{p}{p-1} \ell\left(\varphi\left(\Gamma_{j, k-1}\right)\right)^{p} \\
& \lesssim \sum_{k=1}^{2^{j}} \ell\left(\varphi\left(\Gamma_{j, k}\right)\right)^{p}
\end{aligned}
$$

and

$$
\begin{aligned}
\sum_{k=1}^{2^{j}} \ell\left(\varphi\left(\Gamma_{j, k}\right)\right)^{p-1} \ell\left(\varphi\left(\Gamma_{j, k+1}\right)\right) & \leq \sum_{k=1}^{2^{j}} \frac{1}{p} \ell\left(\varphi\left(\Gamma_{j, k}\right)\right)^{p}+\frac{p}{p-1} \ell\left(\varphi\left(\Gamma_{j, k+1}\right)\right)^{p} \\
& \lesssim \sum_{k=1}^{2^{j}} \ell\left(\varphi\left(\Gamma_{j, k}\right)\right)^{p}
\end{aligned}
$$

Combining (3.37), (3.38) with (3.39) implies that

$$
\left(\int_{\mathbb{S}^{1}} \mathcal{L}^{1}\left(E_{2^{1-j}}(\xi)\right)^{\frac{1}{p-1}}|d \xi|\right)^{p-1} \lesssim \sum_{k=1}^{2^{j}} \ell\left(\varphi\left(\Gamma_{j, k}\right)\right)^{p}
$$

for all $j \in \mathbb{N}$. Let

$$
\Lambda_{\lambda}(t)= \begin{cases}t^{\lambda+1} & \lambda \neq-1 \\ \log t & \lambda=-1\end{cases}
$$

For any $j \in \mathbb{N}$, we have that

$$
\int_{2^{-j}}^{2^{1-j}} t^{-1} \log _{2}^{\lambda}\left(t^{-1}\right) d t \approx-\int_{2^{-j}}^{2^{1-j}} d \Lambda_{\lambda}\left(\log _{2}\left(t^{-1}\right)\right)=\Lambda_{\lambda}(j)-\Lambda_{\lambda}(j-1) \approx j^{\lambda} .
$$

It follows (3.41) and (2.3) that

$$
\int_{2^{-j}}^{2^{1-j}}-\mathcal{A}_{p, \alpha, \lambda}^{\prime}(t) d t \approx 2^{j(p-2-\alpha)} \int_{2^{-j}}^{2^{1-j}} \frac{1}{t} \log _{2}^{\lambda}\left(t^{-1}\right) d t \approx \ell\left(\Gamma_{j, k}\right)^{2+\alpha-p} j^{\lambda}
$$

By combining (3.36), (3.40) with (3.42), we conclude that

$$
\mathcal{V}^{\frac{1}{p-1}}(p, \alpha, \lambda, \varphi) \lesssim \sum_{j=1}^{+\infty} \sum_{k=1}^{2^{j}} \ell\left(\varphi\left(\Gamma_{j, k}\right)\right)^{p} \ell\left(\Gamma_{j, k}\right)^{2+\alpha-p} j^{\lambda}=\mathcal{E}_{1}(p, \alpha, \lambda, \varphi)
$$


We next consider the case $p \in[2,+\infty)$. By the analogous arguments as for (3.36) we have that

$$
\mathcal{V}^{\frac{1}{p-1}}(p, \alpha, \lambda, \varphi) \gtrsim \sum_{j=5}^{+\infty} \int_{\pi 2^{1-j}}^{\pi 2^{2-j}}-\mathcal{A}_{p, \alpha, \lambda}^{\prime}(t) d t\left(\int_{\mathbb{S}^{1}} \mathcal{L}^{1}\left(E_{\pi 2^{1-j}}(\xi)\right)^{\frac{1}{p-1}}|d \xi|\right)^{p-1} .
$$

Since $\varphi\left(\Gamma_{j, k}\right) \subset E_{\pi 2^{1-j}}(\xi)$ for all $j \geq 5, k \in\left\{1, \ldots, 2^{j}\right\}$ and all $\xi \in \varphi\left(\Gamma_{j, k}\right)$, we have that

$$
\begin{aligned}
\left(\int_{\mathbb{S}^{1}} \mathcal{L}^{1}\left(E_{\pi 2^{1-j}}(\xi)\right)^{\frac{1}{p-1}}|d \xi|\right)^{p-1} & =\left(\sum_{k=1}^{2^{j}} \int_{\varphi\left(\Gamma_{j, k}\right)} \mathcal{L}^{1}\left(E_{\pi 2^{1-j}}(\xi)\right)^{\frac{1}{p-1}}|d \xi|\right)^{p-1} \\
& \geq\left(\sum_{k=1}^{2^{j}} \ell\left(\varphi\left(\Gamma_{j, k}\right)\right) \ell\left(\varphi\left(\Gamma_{j, k}\right)\right)^{\frac{1}{p-1}}\right)^{p-1} \\
& \geq \sum_{k=1}^{2^{j}} \ell\left(\varphi\left(\Gamma_{j, k}\right)\right)^{p}
\end{aligned}
$$

By (3.42), (3.43) and (3.44), there is a constant $C>0$ such that

$$
\mathcal{E}_{1}(p, \alpha, \lambda, \varphi)=\sum_{j=1}^{4} \sum_{k=1}^{2^{j}}+\sum_{j=5}^{+\infty} \sum_{k=1}^{2^{j}} \lesssim C+\mathcal{V}^{\frac{1}{p-1}}(p, \alpha, \lambda, \varphi)
$$

We next prove Theorem $3.1(2)$.

Lemma 3.6. Let $\varphi: \mathbb{S}^{1} \rightarrow \mathbb{S}^{1}$ be a homeomorphism. For any $p \in(1,+\infty), \alpha \in$ $(-1, p-1)$ and $\lambda \in \mathbb{R}$, we have that $\mathcal{U}(p, \alpha, \lambda, \varphi)$ and $\mathcal{E}_{1}(p, \alpha, \lambda, \varphi)$ are comparable.

Proof. We first prove that $\mathcal{U}(p, \alpha, \lambda, \varphi)$ is controlled by $\mathcal{E}_{1}(p, \alpha, \lambda, \varphi)$. Given $\xi \in$ $\mathbb{S}^{1}$ and $\eta \in \mathbb{S}^{1}$, let $\ell(\xi \eta)$ be the arc length of the shorter arc in $\mathbb{S}^{1}$ connecting $\xi$ and $\eta$. Given $j \geq 1$ and $\xi \in \mathbb{S}^{1}$, set

$$
A_{j}=\left\{(\xi, \eta) \in \mathbb{S}^{1} \times \mathbb{S}^{1}: \pi 2^{-j}<\ell(\xi \eta) \leq \pi 2^{1-j}\right\}
$$

and $A_{j}(\xi)=\left\{\eta \in \mathbb{S}^{1}:(\xi, \eta) \in A_{j}\right\}$. Notice that $\lambda_{\mathbb{D}}$ is the Euclidean distance. We have that

$$
\begin{aligned}
\mathcal{U}(p, \alpha, \lambda, \varphi) & =\sum_{j=1}^{+\infty} \int_{A_{j}} \Phi_{p, \lambda}\left(\frac{|\varphi(\xi)-\varphi(\eta)|}{|\xi-\eta|}\right)|\xi-\eta|^{\alpha}|d \eta||d \xi| \\
& =\sum_{j=1}^{+\infty} \sum_{k=1}^{2^{j}} \int_{\Gamma_{j, k}} \int_{A_{j}(\xi)} \Phi_{p, \lambda}\left(\frac{|\varphi(\xi)-\varphi(\eta)|}{|\xi-\eta|}\right)|\xi-\eta|^{\alpha}|d \eta||d \xi| .
\end{aligned}
$$


Notice that

$$
|\xi-\eta| \approx \ell\left(\Gamma_{j, k}\right) \text { and }|\varphi(\xi)-\varphi(\eta)| \leq \sum_{i=k-1}^{k+1} \ell\left(\varphi\left(\Gamma_{j, i}\right)\right) \leq 2 \pi
$$

for all $j \in \mathbb{N}, k \in\left\{1, \ldots, 2^{j}\right\}, \xi \in \Gamma_{j, k}$ and $\eta \in A_{j}(\xi)$. It then follows that

$$
\begin{aligned}
\Phi_{p, \lambda}\left(\frac{|\varphi(\xi)-\varphi(\eta)|}{|\xi-\eta|}\right)|\xi-\eta|^{\alpha} & \lesssim\left(\sum_{i=k-1}^{k+1} \ell\left(\varphi\left(\Gamma_{j, i}\right)\right)\right)^{p} \ell\left(\Gamma_{j, k}\right)^{\alpha-p} \log ^{\lambda}\left(e+2 \pi \cdot 2^{j}\right) \\
& \lesssim \sum_{i=k-1}^{k+1} \ell\left(\varphi\left(\Gamma_{j, i}\right)\right)^{p} \ell\left(\Gamma_{j, k}\right)^{\alpha-p} j^{\lambda}
\end{aligned}
$$

for all $\lambda \geq 0, \xi \in \Gamma_{j, k}$ and $\eta \in A_{j}(\xi)$. Since

$$
\mathcal{L}^{1}\left(A_{j}(\xi)\right) \approx \ell\left(\Gamma_{j, k}\right)
$$

for all $j \in \mathbb{N}, k=1, \ldots, 2^{j}$ and $\xi \in \Gamma_{j, k}$, we derive from (3.45) and (3.47) that

$$
\begin{aligned}
\mathcal{U}(p, \alpha, \lambda, \varphi) & \lesssim \sum_{j=1}^{+\infty} \sum_{k=1}^{2^{j}} \sum_{i=k-1}^{k+1} \ell\left(\varphi\left(\Gamma_{j, i}\right)\right)^{p} \ell\left(\Gamma_{j, k}\right)^{\alpha-p} j^{\lambda} \int_{\Gamma_{j, k}} \int_{A_{j}(\xi)}|d \eta||d \xi| \\
& \lesssim \sum_{j=1}^{+\infty} \sum_{k=1}^{2^{j}} \ell\left(\varphi\left(\Gamma_{j, k}\right)\right)^{p} \ell\left(\Gamma_{j, k}\right)^{2+\alpha-p} j^{\lambda}=\mathcal{E}_{1}(p, \alpha, \lambda, \varphi)
\end{aligned}
$$

whenever $\lambda \geq 0$.

Since $\varphi$ is homeomorphic, for any $j \in \mathbb{N}$ and $k \in\left\{1, \ldots, 2^{j}\right\}$ there are $\xi_{j, k}^{\prime} \in \Gamma_{j, k}$ and $\eta_{j, k}^{\prime} \in A_{j}\left(\xi_{j, k}^{\prime}\right)$ such that

$$
\begin{aligned}
& \Phi_{p, \lambda}\left(\frac{\left|\varphi\left(\xi_{j, k}^{\prime}\right)-\varphi\left(\eta_{j, k}^{\prime}\right)\right|}{\left|\xi_{j, k}^{\prime}-\eta_{j, k}^{\prime}\right|}\right)\left|\xi_{j, k}^{\prime}-\eta_{j, k}^{\prime}\right|^{\alpha} \\
= & \max \left\{\Phi_{p, \lambda}\left(\frac{|\varphi(\xi)-\varphi(\eta)|}{|\xi-\eta|}\right)|\xi-\eta|^{\alpha}: \xi \in \Gamma_{j, k} \text { and } \eta \in A_{j}(\xi)\right\} .
\end{aligned}
$$

Since $0<\alpha+1<p$, there is $\beta \in(-1,0)$ such that $0<(1+\beta) p<\alpha+1$. Define

$$
\chi(j, k)= \begin{cases}1 & \text { if }\left|\varphi\left(\xi_{j, k}^{\prime}\right)-\varphi\left(\eta_{j, k}^{\prime}\right)\right| \leq 2^{j \beta}, \\ 0 & \text { otherwise. }\end{cases}
$$


From (3.45), (3.49), (3.48), (2.3) and (3.46), we obtain that

$$
\begin{aligned}
\mathcal{U}(p, \alpha, \lambda, \varphi) & \leq \sum_{j=1}^{+\infty} \sum_{k=1}^{2^{j}} \ell\left(\Gamma_{j, k}\right)^{2+\alpha} \Phi_{p, \lambda}\left(\frac{\left|\varphi\left(\xi_{j, k}^{\prime}\right)-\varphi\left(\eta_{j, k}^{\prime}\right)\right|}{\left|\xi_{j, k}^{\prime}-\eta_{j, k}^{\prime}\right|}\right) \\
& =\sum_{j=1}^{+\infty} \sum_{k=1}^{2^{j}} \ell\left(\Gamma_{j, k}\right)^{2+\alpha} \Phi_{p, \lambda}\left(\frac{\left|\varphi\left(\xi_{j, k}^{\prime}\right)-\varphi\left(\eta_{j, k}^{\prime}\right)\right|}{\left|\xi_{j, k}^{\prime}-\eta_{j, k}^{\prime}\right|}\right) \chi(j, k)
\end{aligned}
$$

$$
+\sum_{j=1}^{+\infty} \sum_{k=1}^{2^{j}} \ell\left(\Gamma_{j, k}\right)^{2+\alpha} \Phi_{p, \lambda}\left(\frac{\left|\varphi\left(\xi_{j, k}^{\prime}\right)-\varphi\left(\eta_{j, k}^{\prime}\right)\right|}{\left|\xi_{j, k}^{\prime}-\eta_{j, k}^{\prime}\right|}\right)(1-\chi(j, k))=: \sum_{1}+\sum_{2} .
$$

Since $\log ^{\lambda}\left(e+\left|\varphi\left(\xi_{j, k}^{\prime}\right)-\varphi\left(\eta_{j, k}^{\prime}\right)\right|\left|\xi_{j, k}^{\prime}-\eta_{j, k}^{\prime}\right|^{-1}\right) \leq 1$ for all $\lambda<0, j \in \mathbb{N}$ and $1 \leq k \leq 2^{j}$, by (3.46) and (2.3) we have that

$$
\sum_{1} \lesssim \sum_{j=1}^{+\infty} 2^{-2 j} \sum_{k=1}^{2^{j}} 2^{j((1+\beta) p-\alpha)}=\sum_{j=1}^{+\infty} 2^{j((1+\beta) p-\alpha-1)}<+\infty
$$

Moreover we derive from (3.46) that

$$
\begin{aligned}
\sum_{2} & \lesssim \sum_{j=1}^{+\infty} \sum_{k=1}^{2^{j}} \ell\left(\Gamma_{j, k}\right)^{2+\alpha-p}\left(\sum_{i=k-1}^{k+1} \ell\left(\varphi\left(\Gamma_{j, k}\right)\right)\right)^{p} \log ^{\lambda}\left(2^{j(1+\beta)}\right) \\
& \lesssim \sum_{j=1}^{+\infty} \sum_{k=1}^{2^{j}} \ell\left(\varphi\left(\Gamma_{j, k}\right)\right)^{p} \ell\left(\Gamma_{j, k}\right)^{2+\alpha-p} j^{\lambda}=\mathcal{E}_{1}(p, \alpha, \lambda, \varphi) .
\end{aligned}
$$

for all $\lambda<0$. Combining (3.50), (3.51) with (3.52) implies that there is a constant $C>0$ such that $\mathcal{U}(p, \alpha, \lambda, \varphi) \lesssim C+\mathcal{E}_{1}(p, \alpha, \lambda, \varphi)$ for all $\lambda<0$.

We next prove that $\mathcal{U}(p, \alpha, \lambda, \varphi)$ dominates $\mathcal{E}_{1}(p, \alpha, \lambda, \varphi)$. Given $j \geq 3$ and $\xi \in \mathbb{S}^{1}$, set

$$
B_{j}=\left\{(\xi, \eta) \in \mathbb{S}^{1} \times \mathbb{S}^{1}: \pi 2^{2-j}<\ell(\xi \eta) \leq \pi 2^{3-j} \text { with } \arg \eta>\arg \xi\right\}
$$

and $B_{j}(\xi)=\left\{\eta \in \mathbb{S}^{1}:(\xi, \eta) \in B_{j}\right\}$. We have that

$$
\sum_{j=3}^{+\infty} \sum_{k=1}^{2^{j}} \int_{\Gamma_{j, k-1}} \int_{B_{j}(\xi)} \Phi_{p, \lambda}\left(\frac{|\varphi(\xi)-\varphi(\eta)|}{|\xi-\eta|}\right)|\xi-\eta|^{\alpha}|d \eta||d \xi|=\mathcal{U}(p, \alpha, \lambda, \varphi)
$$


Since $\varphi$ is homeomorphic, for any $j \geq 3$ and $1 \leq k \leq 2^{j}$ there are $\xi_{j, k}^{\prime \prime} \in \Gamma_{j, k-1}$ and $\eta_{j, k}^{\prime \prime} \in B_{j}\left(\xi_{j, k}^{\prime \prime}\right)$ such that

$$
\begin{aligned}
& \Phi_{p, \lambda}\left(\frac{\left|\varphi\left(\xi_{j, k}^{\prime \prime}\right)-\varphi\left(\eta_{j, k}^{\prime \prime}\right)\right|}{\left|\xi_{j, k}^{\prime \prime}-\eta_{j, k}^{\prime \prime}\right|}\right)\left|\xi_{j, k}^{\prime \prime}-\eta_{j, k}^{\prime \prime}\right|^{\alpha} \\
= & \min \left\{\Phi_{p, \lambda}\left(\frac{|\varphi(\xi)-\varphi(\eta)|}{|\xi-\eta|}\right)|\xi-\eta|^{\alpha}: \xi \in \Gamma_{j, k-1} \text { and } \eta \in B_{j}(\xi)\right\} .
\end{aligned}
$$

Notice that

$$
\left|\xi_{j, k}^{\prime \prime}-\eta_{j, k}^{\prime \prime}\right| \approx \ell\left(\Gamma_{j, k}\right) \text { and } 2 \geq\left|\varphi\left(\xi_{j, k}^{\prime \prime}\right)-\varphi\left(\eta_{j, k}^{\prime \prime}\right)\right| \gtrsim \ell\left(\varphi\left(\Gamma_{j, k}\right)\right)
$$

whenever $j \geq 3$ and $k \in\left\{1, \ldots, 2^{j}\right\}$. Since $\mathcal{L}^{1}\left(B_{j}(\xi)\right) \approx \ell\left(\Gamma_{j, k}\right)$ for all $j \geq 3, k=$ $1, \ldots, 2^{j}$ and $\xi \in \mathbb{S}^{1}$, it follows from (3.53), (3.54) and (3.55) that

$$
\sum_{j=3}^{+\infty} \sum_{k=1}^{2^{j}} \ell\left(\Gamma_{j, k}\right)^{2+\alpha} \Phi_{p, \lambda}\left(\frac{\left|\varphi\left(\xi_{j, k}^{\prime \prime}\right)-\varphi\left(\eta_{j, k}^{\prime \prime}\right)\right|}{\left|\xi_{j, k}^{\prime \prime}-\eta_{j, k}^{\prime \prime}\right|}\right) \mid \lesssim \mathcal{U}(p, \alpha, \lambda, \varphi)
$$

Moreover, for any $\lambda \leq 0$ we obtain from (3.55) that

$$
j^{\lambda} \lesssim \log ^{\lambda}\left(e+2^{1+j}\right) \lesssim \log ^{\lambda}\left(e+\frac{\left|\varphi\left(\xi_{j, k}^{\prime \prime}\right)-\varphi\left(\eta_{j, k}^{\prime \prime}\right)\right|}{\left|\xi_{j, k}^{\prime \prime}-\eta_{j, k}^{\prime \prime}\right|}\right)
$$

for all $j \in \mathbb{N}$ and all $k=1, \ldots, 2^{j}$. From (3.55), (3.56) and (3.57), there is a constant $C>0$ such that

$$
\mathcal{E}_{1}(p, \alpha, \lambda, \varphi)=C+\sum_{j=3}^{+\infty} \sum_{k=1}^{2^{j}} \ell\left(\varphi\left(\Gamma_{j, k}\right)\right)^{p} \ell\left(\Gamma_{j, k}\right)^{2+\alpha-p} j^{\lambda} \lesssim C+\mathcal{U}(p, \alpha, \lambda, \varphi)
$$

for all $\lambda \leq 0$. For any $\lambda>0$, by (3.55) and (3.56) there is a constant $C>0$ such that

$$
\sum_{j=1}^{+\infty} \sum_{k=1}^{2^{j}} \ell\left(\varphi\left(\Gamma_{j, k}\right)\right)^{p} \ell\left(\Gamma_{j, k}\right)^{2+\alpha-p} \log ^{\lambda}\left(2^{j} \ell\left(\varphi\left(\Gamma_{j, k}\right)\right)\right) \lesssim C+\mathcal{U}(p, \alpha, \lambda, \varphi) .
$$

Let $\beta$ be same as in (3). Set

$$
\chi_{j, k}= \begin{cases}1 & \text { if } \ell\left(\varphi\left(\Gamma_{j, k}\right)\right) \leq 2^{j \beta} \\ 0 & \text { otherwise }\end{cases}
$$


We have that

$$
\begin{aligned}
\mathcal{E}_{1}(p, \alpha, \lambda, \varphi)= & \sum_{j=1}^{+\infty} \sum_{k=1}^{2^{j}} \ell\left(\varphi\left(\Gamma_{j, k}\right)\right)^{p} \ell\left(\Gamma_{j, k}\right)^{2+\alpha-p} j^{\lambda} \chi_{j, k} \\
& +\sum_{j=1}^{+\infty} \sum_{k=1}^{2^{j}} \ell\left(\varphi\left(\Gamma_{j, k}\right)\right)^{p} \ell\left(\Gamma_{j, k}\right)^{2+\alpha-p} j^{\lambda}\left(1-\chi_{j, k}\right)=: \sum^{1}+\sum^{2} .
\end{aligned}
$$

Moreover

$$
\sum^{1} \leq \sum_{j=1}^{+\infty} 2^{j((1+\beta) p-\alpha-1)} j^{\lambda}<\infty
$$

and

$$
\sum^{2} \lesssim \sum_{j=1}^{+\infty} \sum_{k=1}^{2^{j}} \ell\left(\varphi\left(\Gamma_{j, k}\right)\right)^{p} \ell\left(\Gamma_{j, k}\right)^{2+\alpha-p} \log ^{\lambda}\left(2^{j} \ell\left(\varphi\left(\Gamma_{j, k}\right)\right)\right)
$$

From (3.59), (3.60), (3.61) and (3.58), we have that $\mathcal{U}(p, \alpha, \lambda, \varphi)$ controls $\mathcal{E}_{1}(p, \alpha, \lambda, \varphi)$ whenever $\lambda>0$.

Proof of Theorem 3.1 (2). By Lemma 3.2, Lemma 3.3 and Lemma 3.4, for any $p>1$ we have that both $I_{1}(p, \alpha, \lambda, h)$ and $I_{2}(p, \alpha, \lambda, h)$ are comparable to $\mathcal{E}_{1}(p, \alpha, \lambda, \varphi)$ whenever $\alpha \in(-1, p-1)$ and $\lambda \in \mathbb{R}$. By Lemma 3.6, we hence conclude comparability of both $I_{1}(p, \alpha, \lambda, h)$ and $I_{2}(p, \alpha, \lambda, h)$ with $\mathcal{U}(p, \alpha, \lambda, \varphi)$ for all $p>1, \alpha \in(-1, p-1)$ and every $\lambda \in \mathbb{R}$. By Lemma 3.5, we can dominate $\mathcal{V}(p, \alpha, \lambda, \varphi)$ by either $I_{1}(p, \alpha, \lambda, h)$ or $I_{2}(p, \alpha, \lambda, h)$ whenever $p \in(1,2]$, while both $I_{1}(p, \alpha, \lambda, h)$ and $I_{2}(p, \alpha, \lambda, h)$ are controlled by $\mathcal{V}(p, \alpha, \lambda, \varphi)$ for all $p \in[2,+\infty)$. Moreover from Example 4.2 and Example 4.3, we have that $\mathcal{V}(p, \alpha, \lambda, \varphi)$ is comparable to either $I_{1}(p, \alpha, \lambda, h)$ or $I_{2}(p, \alpha, \lambda, h)$ only when $p=2$.

Towards the proof of Theorem 3.1 (3), we have the following general result.

Lemma 3.7. Let $\Omega \subset \mathbb{R}^{2}$ be a Jordan domain and $\varphi: \mathbb{S}^{1} \rightarrow \partial \Omega$ be a homeomorphism. For any $p>1$, there is no diffeomorphic extension $h: \mathbb{D} \rightarrow \Omega$ of $\varphi$ for which $I_{1}(p, \alpha, \lambda, h)<+\infty$ for either $\alpha \in(-\infty,-1)$ and $\lambda \in \mathbb{R}$ or $\alpha=-1$ and $\lambda \in[-1,+\infty)$; or for which $I_{2}(p, \alpha, \lambda, h)<+\infty$ for some $\alpha \in(-\infty,-1]$ and $\lambda \in \mathbb{R}$.

Proof. Assume that there is a diffeomorphic extension $h: \mathbb{D} \rightarrow \Omega$ of $\varphi$ for which $I_{1}(p, \alpha, \lambda, h)<+\infty$ for either $\alpha \in(-\infty,-1)$ and $\lambda \in \mathbb{R}$ or $\alpha=-1$ and $\lambda \in$ $[-1,+\infty)$. Then $h \in W^{1, p}(\mathbb{D}, \Omega)$. Let

$$
\mathbb{S}_{r}=\left\{\xi \in \mathbb{R}^{2}:|\xi|=r\right\} \text { and } \operatorname{osc}_{\mathbb{S}_{r}} h=\sup \left\{\left|h\left(\xi_{1}\right)-h\left(\xi_{2}\right)\right|: \xi_{1}, \xi_{2} \in \mathbb{S}_{r}\right\} .
$$


By the ACL-property of Sobolev mappings, we have that

$$
\operatorname{osc}_{\mathbb{S}_{r}} h \leq \int_{\mathbb{S}_{r}}|D h(\xi)||d \xi|
$$

for $\mathcal{L}^{1}$-a.e. $r \in[0,1)$. By Jensen's inequality we derive from (3.62) that

$$
\begin{aligned}
\left(\operatorname{osc}_{\mathbb{S}_{r}} h\right)^{p} & \leq\left(\operatorname{osc}_{\mathbb{S}_{r}} h\right)^{p} r^{1-p} \lesssim \int_{\mathbb{S}_{r}}|D h(\xi)|^{p}|d \xi| \\
& =w_{\alpha, \lambda}^{-1}(1-r) \int_{\mathbb{S}_{r}}|D h(\xi)|^{p} w_{\alpha, \lambda}(1-r)|d \xi| .
\end{aligned}
$$

Let $\mathbb{D}_{r}=\left\{z \in \mathbb{R}^{2}:|z|<r\right\}$. Since $h$ is a homeomorphism, we have $\operatorname{osc}_{\mathbb{D}_{r}} h=$ $\operatorname{osc}_{\mathbb{S}_{r}} h$. Hence

$$
\mathrm{Osc}_{\mathbb{S}_{r}} h \text { is increasing with respect to } r \in[0,1) .
$$

Moreover $w_{\alpha, \lambda}(1-r) \approx 2^{-\alpha j} j^{\lambda}$ for all $j \geq 0$ and $r \in\left(1-2^{-j}, 1-2^{-j-1}\right]$. By (3.63), (3.64) and Fubini's theorem, we obtain that

$$
\begin{aligned}
\sum_{j=1}^{+\infty}\left(\operatorname{osc}_{\mathbb{S}_{1-2^{-j}}} h\right)^{p} 2^{-(\alpha+1) j} j^{\lambda} & \leq \sum_{j=1}^{+\infty} \int_{1-2^{-j}}^{1-2^{-j-1}}\left(\operatorname{osc}_{\mathbb{S}_{r}} h\right)^{p} w_{\alpha, \lambda}(1-r) d r \\
& \lesssim \sum_{j=1}^{+\infty} \int_{1-2^{-j}}^{1-2^{-j-1}} \int_{\mathbb{S}_{r}}|D h(\xi)|^{p} w_{\alpha, \lambda}(1-r)|d \xi| d r \\
& =I_{1}(p, \alpha, \lambda, h) .
\end{aligned}
$$

By the assumption at the beginning, we derive from (3.65) that

$$
\sum_{j=1}^{+\infty}\left(\operatorname{osc}_{\mathbb{S}_{1-2^{-j}}} h\right)^{p} 2^{-(\alpha+1) j} j^{\lambda}<+\infty
$$

for either $\alpha<-1$ and $\lambda \in \mathbb{R}$ or $\alpha=-1$ and $\lambda \geq-1$. Hence by (3.64) we have that $\operatorname{osc}_{\mathbb{S}_{1-2}-j} h=0$ for all $j \geq 1$. Therefore there is a constant $C$ such that $h(z)=C$ for all $z \in \mathbb{D}$. This contradicts the homeomorphicity of $h$. We conclude that the assumption at the beginning cannot hold.

We next assume that there is a diffeomorphic extension $h: \mathbb{D} \rightarrow \Omega$ of $\varphi$ for which $I_{2}(p, \alpha, \lambda, h)<+\infty$ for some $\alpha \in(-\infty,-1]$ and $\lambda \in \mathbb{R}$. It is not difficult to see that $h \in W^{1,1}(\mathbb{D}, \Omega)$. We first let $\lambda \geq 0$. Proposition 2.7 shows that $\Phi_{p, \lambda}$ is convex. Analogously to (3.65), we have

$$
\sum_{j=1}^{+\infty} \Phi_{p, \lambda}\left(\frac{\mathrm{osc}_{\mathbb{S}_{1-2^{-j}}} \operatorname{Re} h}{2 \pi}\right) 2^{-(\alpha+1) j} \lesssim I_{2}(p, \alpha, \lambda, h) .
$$


Analogous arguments as below (3.66) imply that there is a contradiction under the above assumption. We next let $\lambda<0$. Proposition 2.9 shows that $\Psi_{p, \lambda}$ is convex. Analogously to (3.67), we obtain from (2.38) that

$$
\begin{aligned}
\sum_{j=1}^{+\infty} \Psi_{p, \lambda}\left(\frac{\mathrm{osc}_{\mathbb{S}_{1-2^{-j}}} \operatorname{Re} h}{2 \pi}\right) 2^{-(\alpha+1) j} & \lesssim \int_{\mathbb{D}} \Psi_{p, \lambda}(|D h(z)|) w_{\alpha, 0}(z) d z \\
& \approx I_{2}(p, \alpha, \lambda, h) .
\end{aligned}
$$

Analogous arguments as below (3.66) imply that there is a contradiction under the above assumption.

Proof of Theorem 1.1. Let $\lambda_{\Omega}$ be the internal distance and $|\cdot|$ be the Euclidean distance. As the proof of [9, Theorem 1] shows that there exist a bi-Lipschitz mapping $g:\left(\mathbb{S}^{1},|\cdot|\right) \rightarrow\left(\partial \Omega, \lambda_{\Omega}\right)$ and a diffeomorphic bi-Lipschitz extension $\tilde{g}:(\mathbb{D},|\cdot|) \rightarrow\left(\Omega, \lambda_{\Omega}\right)$ of $g$. Let $h=\tilde{g} \circ P\left[g^{-1} \circ \varphi\right]$. Then $h: \mathbb{D} \rightarrow \Omega$ is a diffeomorphic extension of $\varphi$. Moreover

$$
\begin{gathered}
I_{1}(p, \alpha, \lambda, h) \approx I_{1}\left(p, \alpha, \lambda, P\left[g^{-1} \circ \varphi\right]\right), I_{2}(p, \alpha, \lambda, h) \approx I_{2}\left(p, \alpha, \lambda, P\left[g^{-1} \circ \varphi\right]\right), \\
\mathcal{U}(p, \alpha, \lambda, \varphi) \approx \mathcal{U}\left(p, \alpha, \lambda, g^{-1} \circ \varphi\right), \mathcal{V}(p, \alpha, \lambda, \varphi) \approx \mathcal{V}\left(p, \alpha, \lambda, g^{-1} \circ \varphi\right) .
\end{gathered}
$$

Hence Theorem 1.1 (1) and (2) follow from Theorem 3.1. By Lemma 3.7, we complete the proof of Theorem 1.1.

\section{Examples}

In this section, we give examples related to Theorem 3.1 (2). We first decompose $[0,1]$. For a given $s \in(0,+\infty)$, let

$$
j_{n}^{s}=\left[2^{\frac{n}{s}}\right]
$$

be the largest integer less than $2^{n / s}$. There is $n_{0}^{s} \geq 1$ such that

$$
2^{-2-j_{n}^{s}} \geq 2^{-j_{n+1}^{s}} \text { and } 2^{-j_{n}^{s}} \leq 4^{-n} \quad \forall n \geq n_{0}^{s}-1 .
$$

Step 1. Let

$$
I_{1}=I_{1,1}=\left(a_{1,1}, a_{1,2}\right) \quad \text { where } a_{1,1}=4^{-1} \text { and } a_{1,2}=1-4^{-1} .
$$

Renumber the elements in $T_{1}=\{0,1\} \cup \partial I_{1}$ as $\left\{b_{1, i_{1}}: i_{1}=1, \ldots, 4\right\}$ such that $b_{1, i_{1}^{\prime}}<b_{1, i_{1}^{\prime \prime}}$ if $i_{1}^{\prime}<i_{1}^{\prime \prime}$. 
Step 2. Let

$$
I_{2,1}=\left(b_{1,1}+4^{-2}, b_{1,2}-4^{-2}\right) \text { and } I_{2,2}=\left(b_{1,3}+4^{-2}, b_{1,4}-4^{-2}\right) .
$$

Set $I_{2}=\cup_{i=1}^{2} I_{2, i}$, and renumber the elements in $T_{2}=T_{1} \cup \partial I_{2}$ as $\left\{b_{2, i_{2}}: i_{2}=\right.$ $1, \ldots, 8\}$ such that $b_{2, i_{2}^{\prime}}<b_{2, i_{2}^{\prime \prime}}$ if $i_{2}^{\prime}<i_{2}^{\prime \prime}$.

After Step (n-1), we have $\left\{I_{n-1, k_{n-1}}: k_{n-1}=1, \ldots, 2^{n-2}\right\}, I_{n-1}=\cup_{k_{n-1}=1}^{2_{-1}} I_{n-1, k_{n-1}}$ and $T_{n-1}:=T_{n-2} \cup \partial I_{n-1}=\left\{b_{n-1, i_{n-1}}: i_{n-1}=1, \ldots, 2^{n}\right\}$ where $b_{n-1, i_{n-1}^{\prime}}<b_{n-1, i_{n-1}^{\prime \prime}}$ if $i_{n-1}^{\prime}<i_{n-1}^{\prime \prime}$. In the following Step n, set

$$
I_{n, k_{n}}:=\left(b_{n-1,2 k_{n}-1}+4^{-n}, b_{n-1,2 k_{n}}-4^{-n}\right) \quad \text { for } k_{n}=1, \ldots, 2^{n-1} .
$$

and $I_{n}=\cup_{k_{n}=1}^{2^{n-1}} I_{n, k_{n}}$. After renumbering the elements in $T_{n}=T_{n-1} \cup \partial I_{n}$ as above, we can proceed to Step $(\mathrm{n}+1)$. Moreover we must replace $I_{n, k_{n}}$ in (4.3) by

$$
I_{n, k_{n}}=\left(b_{n-1,2 k_{n}-1}+2^{-j_{n}^{s}}, b_{n-1,2 k_{n}}-2^{-j_{n}^{s}}\right)
$$

whenever $n \geq n_{0}^{s}$. Let $I=\cup_{n=1}^{\infty} I_{n}$ and $R=[0,1] \backslash I$. Then $R \neq \emptyset$. We finally decompose $[0,1]$ as

$$
R \cup I .
$$

We next give an estimate on the length of $I_{n, k_{n}}$. Since $\mathcal{L}^{1}\left(I_{n, k_{n}}\right)=2^{-j_{n-1}}-2^{1-j_{n}}$ for all $n \geq n_{0}+1$ and $k_{n} \in\left\{1, \ldots, 2^{n-1}\right\}$, by the first inequality in (4.2) we have that

$$
\mathcal{L}^{1}\left(I_{n, k_{n}}\right) \geq 2^{-1-j_{n-1}} .
$$

for all $n \geq n_{0}+1$ and $k_{n} \in\left\{1, \ldots, 2^{n-1}\right\}$. When $n=n_{0}$, from (4.4) and the second estimate in (4.2) we have that

$$
\mathcal{L}^{1}\left(I_{n, k_{n}}\right)=4^{1-n_{0}}-2^{1-j_{n_{0}}} \geq 4^{-n_{0}+1 / 2}>4^{-n_{0}}
$$

for all $k_{n}=1, \ldots, 2^{n-1}$. Whenever $1 \leq n \leq n_{0}-1$ and $k_{n} \in\left\{1, \ldots, 2^{n-1}\right\}$, we have $\mathcal{L}^{1}\left(I_{n, k_{n}}\right)=4^{-n}$. Let $C_{1}(s)=\min \left\{2^{j_{n-1}-2 n}: 1 \leq n \leq n_{0}\right\}$. Then

$$
\mathcal{L}^{1}\left(I_{n, k_{n}}\right) \geq C_{1}(s) 2^{-j_{n-1}}
$$

for all $1 \leq n \leq n_{0}$ and $k_{n} \in\left\{1, \ldots, 2^{n-1}\right\}$. By (4.6), (4.7) and (4.8), we obtain that there is a constant $C(s)>0$ such that

$$
\mathcal{L}^{1}\left(I_{n, k_{n}}\right) \geq C(s) 2^{-j_{n-1}}
$$

for all $n \in \mathbb{N}$ and $k_{n} \in\left\{1, \ldots, 2^{n-1}\right\}$. 
Define

$$
f_{n, s}^{1}(x)=\sum_{k_{n}=1}^{2^{n-1}} \frac{2 k_{n}-1}{2^{n}} \chi_{\overline{I_{n, k_{n}}}}(x) \text { and } f_{s}^{1}(x)=\sum_{n=1}^{+\infty} f_{n, s}^{1}(x) .
$$

For any $x \in R$ and any $n \geq n_{0}^{s}$, there is $b_{n} \in \partial I_{n}$ such that $\left|b_{n}-x\right|=\inf _{b \in \partial I_{n}}|b-x|$. By (4.4) and (4.10), we have that

$$
\left|b_{n}-x\right| \leq 2^{-j_{n}} \text { and }\left|f_{s}^{1}\left(b_{n+1}\right)-f_{s}^{1}\left(b_{n}\right)\right|<2^{-n-1} .
$$

It follows that $\lim _{n \rightarrow+\infty} b_{n}=x$ and $\left\{f^{1}\left(b_{n}\right)\right\}$ is a Cauchy sequence. Therefore

$$
f_{s}(x)= \begin{cases}f_{s}^{1}(x) & \text { if } x \in I \\ \lim _{n \rightarrow+\infty} f_{s}^{1}\left(b_{n}\right) & \text { if } x \in R\end{cases}
$$

is a well-defined function on $[0,1]$.

Proposition 4.1. Let $f_{s}$ be as in (4.11) with $s \in(0,+\infty)$. Then $f_{s}(0)=0$, $f_{s}(1)=1$ and $f_{s}$ is increasing on $[0,1]$. Moreover there is a constant $C(s)>0$ such that

$$
|f(x)-f(y)| \log ^{s}\left(|x-y|^{-1}\right) \leq C(s)
$$

for all $x, y \in[0,1]$ with $x \neq y$.

Proof. By (4.11), we have that $f_{s}(0)=\lim _{n \rightarrow \infty} f_{s}^{1}\left(2^{-j_{n}}\right)=\lim _{n \rightarrow \infty} 2^{-n}=0$. Analogously $f_{s}(1)=1$.

We next prove the monotonicity of $f_{s}$. Let $x_{1} \in[0,1], x_{2} \in[0,1]$ with $x_{1} \leq x_{2}$. If $x_{1} \in I_{n, k_{n}^{\prime}}$ and $x_{2} \in I_{n, k_{n}^{\prime \prime}}$ with $k_{n}^{\prime} \leq k_{n}^{\prime \prime}$, from (4.11) we have that

$$
f_{s}\left(x_{1}\right) \leq f_{s}\left(x_{2}\right) .
$$

Assume $x_{1} \in I_{n_{1}, k_{n_{1}}}$ and $x_{2} \in I_{n_{2}, k_{n_{2}}}$ with $n_{1} \neq n_{2}$. Let $q=\left|n_{2}-n_{1}\right|$. If $n_{1}<n_{2}$, from the construction of $\left\{I_{n, k_{n}}\right\}$ we have that $k_{n_{2}} \geq 2^{q}\left(k_{n_{1}}-1\right)+2^{q-1}+1$. It then follows from (4.10) that

$$
f_{s}\left(x_{2}\right) \geq \frac{2\left(2^{q}\left(k_{n_{1}}-1\right)+2^{q-1}+1\right)-1}{2^{n_{1}} 2^{q}}>f_{s}\left(x_{1}\right) .
$$

If $n_{2}<n_{1}$, from the construction of $\left\{I_{n, k_{n}}\right\}$ we have that

$$
k_{n_{2}} \geq \begin{cases}{\left[\frac{k_{n_{1}}}{2^{q}}\right]+1 \quad \text { if } 0 \leq \frac{k_{n_{1}}}{2^{q}}-\left[\frac{k_{n_{1}}}{2^{q}}\right] \leq 1 / 2,} \\ {\left[\frac{k_{n_{1}}}{2^{q}}\right]+2 \quad \text { if } 1 / 2<\frac{k_{n_{1}}}{2^{q}}-\left[\frac{k_{n_{1}}}{2^{q}}\right]<1}\end{cases}
$$


It follows that

$$
2 k_{n_{2}}-1 \geq 2\left(\frac{k_{n_{1}}}{2^{q}}+1 / 2\right)-1=2 \frac{k_{n_{1}}}{2^{q}} \quad \text { if } 0 \leq \frac{k_{n_{1}}}{2^{q}}-\left[\frac{k_{n_{1}}}{2^{q}}\right] \leq 1 / 2
$$

and

$$
2 k_{n_{2}}-1 \geq 2\left(\frac{k_{n_{1}}}{2^{q}}+1\right)-1=2 \frac{k_{n_{1}}}{2^{q}}+1 \quad \text { if } 1 / 2<\frac{k_{n_{1}}}{2^{q}}-\left[\frac{k_{n_{1}}}{2^{q}}\right]<1 .
$$

By combining (4.15) with (4.16), we deduce from (4.10) that

$$
f_{s}\left(x_{2}\right)>f_{s}\left(x_{1}\right) .
$$

Assume $x_{1} \in R$ and $x_{2} \in I$. By (4.11), there is $\left\{b_{n}\right\} \subset \partial I$ such that $\lim _{n \rightarrow \infty} b_{n}=x_{1}$. Together with $x_{1}<x_{2}$, it follows that $b_{n}<x_{2}$ whenever $n \gg 1$. Via the arguments for (4.13), (4.14) and (4.17), we have that

$$
f_{s}^{1}\left(b_{n}\right) \leq f_{s}\left(x_{2}\right) \quad \forall n \gg 1 .
$$

By taking limit for (4.18), we have that

$$
f_{s}\left(x_{1}\right) \leq f_{s}\left(x_{2}\right)
$$

Assume either $x_{1} \in I$ and $x_{2} \in R$, or $x_{1} \in R$ and $x_{2} \in R$. Via analogous arguments as for (4.19), we can also prove $f_{s}\left(x_{1}\right) \leq f_{s}\left(x_{2}\right)$ at these two cases. By preceding arguments, we conclude that $f_{s}$ is increasing on $[0,1]$.

We next prove (4.12). Let $T_{n}=\left\{b_{n, i_{n}}: i_{n}=1, \ldots, 2^{n+1}\right\}$ with $n \in \mathbb{N}$ and $f_{i, s}^{1}$ be as in (4.10). For a given $n \in \mathbb{N}$, define

$$
\begin{gathered}
f_{n, s}^{2}(x)=\sum_{i=1}^{2^{n}}\left(\frac{2^{j_{n}}}{2^{n}}\left(x-b_{n, 2 i-1}\right)+\frac{i-1}{2^{n}}\right) \chi_{\left[b_{n, 2 i-1}, b_{n, 2 i}\right]}(x), \\
f_{n, s}(x)=f_{n, s}^{2}(x)+\sum_{i=1}^{n} f_{i, s}^{1}(x) .
\end{gathered}
$$

Then $f_{n, s}$ is piecewise affine, increasing and continuous on $[0,1]$. Furthermore we claim:

(i) $\lim _{n \rightarrow \infty} f_{n, s}\left(x_{0}\right)=f_{s}\left(x_{0}\right)$ for all $x_{0} \in[0,1]$, 
(ii) there are constant $C(s)>0$ and $N(s)>0$ such that

$$
\sup \left\{\left|f_{n, s}(x)-f_{n, s}(y)\right| \log ^{s}\left(|x-y|^{-1}\right): x, y \in[0,1] \text { and } x \neq y\right\} \leq C(s)
$$

for all $n \geq N(s)$.

If both (i) and (ii) hold, we can prove (4.12).

We first prove (i). Let $x_{0} \in[0,1]$. If $x_{0} \in I$, without loss of generality we assume that $x_{0} \in I_{n_{0}, k_{n_{0}}}$. From (4.11) and (4.20), we have that $f_{n}\left(x_{0}\right)=f\left(x_{0}\right)$ for all $n \geq n_{0}$. Therefore (i) holds. If $x_{0} \in R$, from (4.11) there is $\left\{b_{n}\right\} \subset \partial I$ such that $\lim _{n \rightarrow \infty} b_{n}=x_{0}$ and $\lim _{n \rightarrow \infty} f_{s}^{1}\left(b_{n}\right)=f_{s}\left(x_{0}\right)$. Moreover by (4.20), we have that

$$
\left|f_{n, s}\left(x_{0}\right)-f_{s}^{1}\left(b_{n}\right)\right|=\left|f_{n, s}\left(x_{0}\right)-f_{n, s}\left(b_{n}\right)\right| \leq 2^{-n} .
$$

Together with $\left|f_{n, s}\left(x_{0}\right)-f_{s}\left(x_{0}\right)\right| \leq\left|f_{n, s}\left(x_{0}\right)-f_{s}^{1}\left(b_{n}\right)\right|+\left|f_{s}^{1}\left(b_{n}\right)-f_{s}\left(x_{0}\right)\right|$, we have that (i) also holds at this case.

We next prove (ii). Given $n \geq 1, x \in[0,1]$ and $y \in[0,1]$ with $x<y$, set

$$
k_{n}(x, y)=\#\left\{I_{m, k_{m}}: I_{m, k_{m}} \subset[x, y] \text { for } m=1, \ldots, n \text { and } k_{m}=1, \ldots, 2^{m-1}\right\} \text {. }
$$

Then $0 \leq k_{n}(x, y) \leq 2^{n}-1$.

Assume $k_{n}(x, y)=0$. If $x \in \cup_{m=1}^{n} I_{m}$, there are $m \in\{1, \ldots, n\}$ and $k_{m} \in$ $\left\{1, \ldots, 2^{m-1}\right\}$ such that $x \in I_{m, k_{m}}$. For the location of $y$, possibly we have that

$$
y \in I_{m, m_{k}}, y \in I_{m, m_{k}+1} \text {, or } y \in[0,1] \backslash\left(\cup_{m=1}^{n} I_{m}\right) .
$$

If $y \in I_{m, m_{k}}$, by (4.20) we have that

$$
f_{n, s}(x)=f_{n, s}(y) \quad \forall n \geq m .
$$

If $y \in I_{m, m_{k}+1}$, then $|x-y| \geq 2^{-j_{n}}$. It follows from (4.20) that

$$
\left|f_{n, s}(x)-f_{n, s}(y)\right| \log ^{s}\left(|x-y|^{-1}\right) \leq 2^{-n} \log ^{s}\left(2^{j_{n}}\right)<1 .
$$

If $y \in[0,1] \backslash\left(\cup_{m=1}^{n} I_{m}\right)$, there is $x_{0} \in[x, y) \cap T_{n}$ such that

$$
0<y-x_{0}<2^{-j_{n}} \text { and } f_{n, s}(x)=f_{n, s}\left(x_{0}\right) .
$$

Since there is $n_{1}^{s}>0$ such that $\log \left(2^{j_{n}^{s} s}\right)-s>0$, we have that

$$
t \log ^{s}\left(t^{-1}\right) \leq 2^{-j_{n}^{s}} \log ^{s}\left(2^{j_{n}^{s}}\right)<2^{n-j_{n}}
$$


for all $n \geq n_{1}^{s}$ and every $t \in\left(0,2^{-j_{n}^{s}}\right]$. By (4.20), (4.23) and (4.24), we then have that

$$
\begin{aligned}
\left|f_{n, s}(x)-f_{n, s}(y)\right| \log ^{s}\left(|x-y|^{-1}\right) & \leq \frac{\left|f_{n, s}\left(x_{0}\right)-f_{n, s}(y)\right|}{\left|x_{0}-y\right|}\left|x_{0}-y\right| \log ^{s}\left(\left|x_{0}-y\right|^{-1}\right) \\
& <\frac{2^{j_{n}}}{2^{n}} \frac{2^{n}}{2^{j_{n}}}=1
\end{aligned}
$$

whenever $n \geq N(s):=\max \left\{n_{0}^{s}, n_{1}^{s}\right\}$. If $x \in[0,1] \backslash\left(\cup_{m=1}^{n} I_{m}\right)$, for the location of $y$ we possibly have that

$$
y \in[0,1] \backslash\left(\cup_{m=1}^{n} I_{m}\right), y \in \cup_{m=1}^{n} I_{m} .
$$

If $y \in[0,1] \backslash\left(\cup_{m=1}^{n} I_{m}\right)$, then $0<y-x<2^{-j_{n}}$. By (4.20) and (4.24) we have that

$$
\left|f_{n, s}(x)-f_{n, s}(y)\right| \log ^{s}\left(|x-y|^{-1}\right)=\frac{2^{j_{n}}}{2^{n}}|x-y| \log ^{s}\left(|x-y|^{-1}\right)<1
$$

for all $n \geq N(s)$. If $y \in \cup_{m=1}^{n} I_{m}$, by analogous arguments as for (4.25) we have that

$$
\left|f_{n, s}(y)-f_{n, s}(x)\right| \log ^{s}\left(|x-y|^{-1}\right)<1
$$

for all $n \geq N(s)$. By (4), (4.22), (4.25), (4.26) and (4.27), we conclude that

$$
\left|f_{n, s}(y)-f_{n, s}(x)\right| \log ^{s}\left(|x-y|^{-1}\right)<1
$$

for all $n \geq N(s)$ and $k_{n}(x, y)=0$.

Assume $k_{n}(x, y) \in\left\{1, \ldots, 2^{n}-1\right\}$. Define

$$
x^{\prime}=\inf \left\{e \in I_{m, k_{m}}: I_{m, k_{m}} \subset[x, y] \text { for } m=1, \ldots, n \text { and } k_{m}=1, \ldots, 2^{m-1}\right\}
$$

and

$$
y^{\prime}=\sup \left\{e \in I_{m, k_{m}}: I_{m, k_{m}} \subset[x, y] \text { for } m=1, \ldots, n \text { and } k_{m}=1, \ldots, 2^{m-1}\right\} .
$$

If $k_{n}(x, y)=1$, by $(4.20)$ we have that

$$
f_{n, s}\left(x^{\prime}\right)=f_{n, s}\left(y^{\prime}\right)
$$

If $2^{m} \leq k_{n}(x, y) \leq 2^{m+1}-1$ for $m=1, \ldots, n-1$, by (4.5), (4.9) and (4.20) we have that

$$
|x-y| \geq \mathcal{L}^{1}\left(I_{n-m, k_{n-m}}\right) \geq C(s) 2^{-j_{n-m-1}^{s}}
$$


and

$$
\left|f_{n, s}\left(x^{\prime}\right)-f_{n, s}\left(y^{\prime}\right)\right|=\frac{2+\ldots+2^{m}}{2^{n}}<2^{m+1-n} .
$$

Whenever $n \geq n_{0}^{s}+1$, it follows from (4.1) that

$$
\begin{aligned}
\left|f_{n, s}\left(x^{\prime}\right)-f_{n, s}\left(y^{\prime}\right)\right| \log ^{s}\left(|x-y|^{-1}\right) & \leq 2^{m+1-n} \log ^{s}\left(C^{-1} 2^{j_{n-m-1}^{s}}\right) \\
& \leq C(s) 2^{m+1-n} j_{n-m-1}^{s}<C(s) .
\end{aligned}
$$

Notice that there are two cases for the location of $x$

$$
x \in\left(x^{\prime}-2^{-j_{n}}, x^{\prime}\right], x \in \cup_{m=1}^{n} I_{m} .
$$

If $x \in\left(x^{\prime}-2^{-j_{n}}, x^{\prime}\right]$, by analogous arguments as for (4.26) we have that

$$
\left|f_{n, s}(x)-f_{n, s}\left(x^{\prime}\right)\right| \log ^{s}\left(|x-y|^{-1}\right)<1 \quad \text { whenever } n \geq N(s) .
$$

If $x \in \cup_{m=1}^{n} I_{m}$, same arguments as (4.25) imply (4.31). Analogously, we have that

$$
\left|f_{n, s}\left(y^{\prime}\right)-f_{n, s}(y)\right| \log ^{s}\left(|x-y|^{-1}\right)<1 \quad \text { whenever } n \geq N(s) .
$$

Since

$$
\begin{aligned}
& \left|f_{n, s}(x)-f_{n, s}(y)\right| \log ^{s}\left(|x-y|^{-1}\right) \\
= & \left(\left|f_{n, s}(x)-f_{n, s}\left(x^{\prime}\right)\right|+\left|f_{n, s}\left(x^{\prime}\right)-f_{n, s}\left(y^{\prime}\right)\right|+\left|f_{n, s}\left(y^{\prime}\right)-f_{n, s}(y)\right|\right) \log ^{s}\left(|x-y|^{-1}\right),
\end{aligned}
$$

by (4.29), (4.30), (4.31) and (4.32) there is a constant $C(s)>0$ such that

$$
\left|f_{n, s}(x)-f_{n, s}(y)\right| \log ^{s}\left(|x-y|^{-1}\right) \leq C(s)
$$

whenever $n \geq N(s)$ and $k_{n}(x, y) \in\left\{1, \ldots, 2^{n}-1\right\}$. By (4.28) and (4.33), we finish the proof of (ii).

Let $\varphi: \mathbb{S}^{1} \rightarrow \mathbb{S}^{1}$ be a homeomorphism. In the following we denote by $P[\varphi]$ : $\mathbb{D} \rightarrow \mathbb{D}$ the harmonic extension of $\varphi$.

Example 4.2. For a given $p \in(1,2)$, there is a homeomorphism $\varphi: \mathbb{S}^{1} \rightarrow \mathbb{S}^{1}$ such that $\mathcal{V}(p, p-2,0, \varphi)<\infty, I_{1}(p, p-2,0, P[\varphi])=\infty$ and $I_{2}(p, p-2,0, P[\varphi])=\infty$.

Proof. We first introduce a class of self-homeomorphisms on $\mathbb{S}^{1}$ and their properties. Let $f_{s}$ be as in (4.11) with $s \in(0,+\infty)$. Define

$$
g_{s}(x)=\frac{f_{s}(x)+x}{2} \quad x \in[0,1] .
$$


Then $g_{s}:[0,1] \rightarrow[0,1]$ is strictly increasing and continuous, i.e. $g_{s}$ is homeomorphic. Moreover by (4.1), there is a constant $C(s)>0$ such that

$$
\left|g_{s}(x)-g_{s}(y)\right| \leq C(s) \log ^{-s}\left(|x-y|^{-1}\right)
$$

for all $x, y \in[0,1]$ with $x \neq y$. Let $\arg z \in(-\pi, \pi]$ be the principal value of the argument $z$. Define

$$
\varphi_{s}(z)=\exp \left(i 2 \pi\left[g_{s}\left(\frac{\arg z}{2 \pi}\right)-g_{s}\left(\frac{1}{2}\right)+\frac{1}{2}\right]\right) \quad z \in \mathbb{S}^{1} .
$$

Then $\varphi_{s}: \mathbb{S}^{1} \rightarrow \mathbb{S}^{1}$ is homeomorphic and $\varphi\left(e^{i \pi}\right)=e^{i \pi}$. Next we prove that

$$
\left|\varphi_{s}\left(z_{1}\right)-\varphi_{s}\left(z_{2}\right)\right| \lesssim \log ^{-s}\left(\left|z_{1}-z_{2}\right|^{-1}\right)
$$

for all $z_{1}, z_{2} \in \mathbb{S}^{1}$ with $z_{1} \neq z_{2}$. Let $\Gamma\left(z_{1}, z_{2}\right)$ be the arc in $\mathbb{S}^{1}$ joining $z_{1}$ to $z_{2}$ with smaller length. Denote by $\ell\left(\Gamma\left(z_{1}, z_{2}\right)\right)$ the length of $\Gamma\left(z_{1}, z_{2}\right)$. In order to prove (4.37), it is enough to consider the case $\ell\left(\Gamma\left(z_{1}, z_{2}\right)\right) \ll 1$. If $e^{i \pi} \notin \Gamma\left(z_{1}, z_{2}\right)$, we have that

$$
\left|\arg z_{1}-\arg z_{2}\right| \approx\left|z_{1}-z_{2}\right| \text { and }\left|g_{s}\left(\frac{\arg z_{1}}{2 \pi}\right)-g_{s}\left(\frac{\arg z_{2}}{2 \pi}\right)\right| \approx\left|\varphi_{s}\left(z_{1}\right)-\varphi_{s}\left(z_{2}\right)\right|
$$

whenever $\ell\left(\Gamma\left(z_{1}, z_{2}\right)\right) \ll 1$. Together with (4.35), we then have that

$$
\begin{aligned}
\left|\varphi_{s}\left(z_{1}\right)-\varphi_{s}\left(z_{2}\right)\right| & \approx\left|g_{s}\left(\frac{\arg z_{1}}{2 \pi}\right)-g_{s}\left(\frac{\arg z_{2}}{2 \pi}\right)\right| \\
& \lesssim \log ^{-s}\left(\left|\arg z_{1}-\arg z_{2}\right|^{-1}\right) \approx \log ^{-s}\left(\left|z_{1}-z_{2}\right|^{-1}\right) .
\end{aligned}
$$

If $e^{i \pi} \in \Gamma\left(z_{1}, z_{2}\right)$ and $\ell\left(\Gamma\left(\varphi\left(z_{1}\right), \varphi\left(e^{i \pi}\right)\right)\right)>\ell\left(\Gamma\left(\varphi\left(e^{i \pi}\right), \varphi\left(z_{2}\right)\right)\right)$, there is $z_{0} \in$ $\Gamma\left(z_{1}, e^{i \pi}\right)$ such that

$$
\left|\varphi_{s}\left(z_{1}\right)-\varphi_{s}\left(z_{2}\right)\right| \lesssim\left|\varphi_{s}\left(z_{1}\right)-\varphi_{s}\left(z_{0}\right)\right|
$$

Same arguments as for (4.38) imply that

$$
\left|\varphi_{s}\left(z_{1}\right)-\varphi_{s}\left(z_{0}\right)\right| \lesssim \log ^{-s}\left(\left|z_{1}-z_{0}\right|^{-1}\right) \lesssim \log ^{-s}\left(\left|z_{1}-z_{2}\right|^{-1}\right) .
$$

Combining (4.39) with (4.40) therefore implies that (4.37) holds when $e^{i \pi} \in$ $\Gamma\left(z_{1}, z_{2}\right)$ and $\ell\left(\Gamma\left(\varphi_{s}\left(z_{1}\right), \varphi_{s}\left(e^{i \pi}\right)\right)\right)>\ell\left(\Gamma\left(\varphi\left(e^{i \pi}\right)\right.\right.$. Analogously, we can prove that (4.37) holds when $e^{i \pi} \in \Gamma\left(z_{1}, z_{2}\right)$ and $\ell\left(\Gamma\left(\varphi_{s}\left(z_{1}\right), \varphi_{s}\left(e^{i \pi}\right)\right)\right) \leq \ell\left(\Gamma\left(\varphi_{s}\left(e^{i \pi}\right), \varphi_{s}\left(z_{2}\right)\right)\right)$.

Let $p \in(1,2)$. There is $s \in(1,+\infty)$ such that $p-1<1 / s<1$. Based on this $s$, we obtain a homeomorphism $\varphi=\varphi_{s}: \mathbb{S}^{1} \rightarrow \mathbb{S}^{1}$, where $\varphi_{s}$ is from (4.36). By 
Jensen's inequality and (4.37), we have that

$$
\begin{aligned}
\mathcal{V}(p, p-2,0, \varphi) & =\int_{\mathbb{S}^{1}}\left(\int_{\mathbb{S}^{1}} \log \left|\varphi^{-1}(\xi)-\varphi^{-1}(\eta)\right|^{-1}|d \eta|\right)^{p-1}|d \xi| \\
& \leq\left(\int_{\mathbb{S}^{1}} \int_{\mathbb{S}^{1}} \log \left|\varphi^{-1}(\xi)-\varphi^{-1}(\eta)\right|^{-1}|d \eta||d \xi|\right)^{p-1} \\
& \lesssim\left(\int_{\mathbb{S}^{1}} \int_{\mathbb{S}^{1}}|\xi-\eta|^{-\frac{1}{s}}|d \eta||d \xi|\right)^{p-1}<+\infty .
\end{aligned}
$$

Let $n_{0}^{s}$ be as in (4.2) with $s$ chosen above. For any $n \geq n_{0}^{s}$ and any $j_{n}<j \leq j_{n+1}$, by (4.34) and (4.11) we have that

$$
\begin{aligned}
\sum_{k=1}^{2^{j}} \ell\left(\varphi\left(\Gamma_{j, k}\right)\right)^{p} & =2 \pi \sum_{k=1}^{2^{j}} \mathcal{L}^{1}\left(g_{s}\left(\left[(k-1) 2^{-j}, k 2^{-j}\right]\right)\right)^{p} \\
& \gtrsim \sum_{k=1}^{2^{j}}\left(f_{s}\left(k 2^{-j}\right)-f_{s}\left((k-1) 2^{-j}\right)\right)^{p}=2^{(n+1)(1-p)} .
\end{aligned}
$$

Notice that $j_{n+1}-j_{n} \approx 2^{n / s}$ whenever $n \geq n_{0}$. We then derive from (4.41) that

$$
\mathcal{E}_{1}(p, p-2,0, \varphi) \geq \sum_{n=n_{0}}^{+\infty} \sum_{j_{n}<j \leq j_{n+1}} \sum_{k=1}^{2^{j}} \ell\left(\varphi\left(\Gamma_{j, k}\right)\right)^{p} \gtrsim \sum_{n=n_{0}}^{+\infty} 2^{n\left(1-p+\frac{1}{s}\right)}=+\infty .
$$

By Lemma 3.2, Lemma 3.3 and Lemma 3.4, it follows that $I_{1}(p, p-2,0, P[\varphi])=\infty$ and $I_{2}(p, p-2,0, P[\varphi])=\infty$.

Example 4.3. For a given $p \in(2,+\infty)$, there is a homeomorphism $\varphi: \mathbb{S}^{1} \rightarrow \mathbb{S}^{1}$ such that $\mathcal{V}(p, p-2,0, \varphi)=\infty, I_{1}(p, p-2,0, P[\varphi])<\infty$ and $I_{2}(p, p-2,0, P[\varphi])<$ $\infty$.

Proof. Since $p \in(2,+\infty)$, there is $s \in(0,1)$ such that $p-1>1 / s>1$. Based on this chosen $s$, we obtain a homeomorphism $\varphi=\varphi_{s}: \mathbb{S}^{1} \rightarrow \mathbb{S}^{1}$, where $\varphi_{s}$ is from (4.36). In order to prove $\mathcal{V}(p, p-2,0, \varphi)=\infty$, by Jensen's inequality it suffices to prove that

$$
\int_{\mathbb{S}^{1}} \int_{\mathbb{S}^{1}} \log \left|\varphi^{-1}(\xi)-\varphi^{-1}(\eta)\right|^{-1}|d \eta||d \xi|=+\infty
$$

For any $\sigma \in \mathbb{S}^{1}$ and $\tau \in \mathbb{S}^{1}$, let $\ell(\sigma, \tau)$ be the arc length of the shorter arc in $\mathbb{S}^{1}$ joining $\sigma$ and $\tau$. Let $n_{0}^{s}$ be from (4.2) with $s$ chosen above. For any $n \geq n_{0}^{s}$, set

$$
\Gamma_{n}=\left\{(\xi, \eta) \in \mathbb{S}^{1} \times \mathbb{S}^{1}: \pi 2^{1-j_{n+1}}<\ell\left(\varphi^{-1}(\xi), \varphi^{-1}(\eta)\right) \leq \pi 2^{1-j_{n}}\right\} .
$$


We have that

$$
\begin{aligned}
\int_{\mathbb{S}^{1}} \int_{\mathbb{S}^{1}} \log \left|\varphi^{-1}(\xi)-\varphi^{-1}(\eta)\right|^{-1}|d \eta||d \xi| & \geq \sum_{n=n_{0}}^{+\infty} \int_{\Gamma_{n}} \log \left|\varphi^{-1}(\xi)-\varphi^{-1}(\eta)\right|^{-1}|d \eta||d \xi| \\
& \gtrsim \sum_{n=n_{0}}^{+\infty} j_{n} \int_{\Gamma_{n}}|d \eta||d \xi| .
\end{aligned}
$$

Given $n \geq n_{0}^{s}$ and $k=1, \ldots, 2^{n}$, let

$$
\begin{gathered}
\Gamma_{n, k}^{\prime}=\exp \left(i 2 \pi\left[b_{n, 2 k-1}, 2^{-j_{n+1}}+b_{n, 2 k-1}\right]\right), \\
\Gamma_{n, k}^{\prime \prime}=\exp \left(i 2 \pi\left[2^{-j_{n}}-2^{-j_{n+1}}+b_{n, 2 k-1}, 2^{-j_{n}}+b_{n, 2 k-1}\right]\right) .
\end{gathered}
$$

For any $\xi \in \varphi\left(\Gamma_{n, k}^{\prime}\right)$ and $\eta \in \varphi\left(\Gamma_{n, k}^{\prime \prime}\right)$, we have that

$$
2 \pi\left(2^{-j_{n}}-2^{1-j_{n+1}}\right) \leq \ell\left(\varphi^{-1}(\xi), \varphi^{-1}(\eta)\right) \leq \pi \cdot 2^{1-j_{n}} .
$$

Notice that by (4.2) we have that $2^{-j_{n+1}}<2^{-j_{n}}-2^{1-j_{n+1}}$ whenever $n \geq n_{0}^{s}$. It then follows from (4.44) that

$$
\varphi\left(\Gamma_{n, k}^{\prime}\right) \times \varphi\left(\Gamma_{n, k}^{\prime \prime}\right) \subset \Gamma_{n}
$$

for all $n \geq n_{0}^{s}$ and all $k=1, \ldots, 2^{n}$. Moreover from (4.36), (4.34) and (4.11), it follows that

$$
\begin{aligned}
\ell\left(\varphi\left(\Gamma_{n, k}^{\prime}\right)\right) & =2 \pi \mathcal{L}^{1}\left(g\left(\left[b_{n, 2 k-1}, 2^{-j_{n+1}}+b_{n, 2 k-1}\right]\right)\right) \\
& \geq \pi\left(f_{s}\left(2^{-j_{n+1}}\right)-f_{s}(0)\right)=\pi 2^{-n-1} .
\end{aligned}
$$

for all $n \geq n_{0}^{s}$ and all $k=1, \ldots, 2^{n}$. Similarly

$$
\ell\left(\varphi\left(\Gamma_{n, k}^{\prime \prime}\right)\right) \geq \pi 2^{-n-1} .
$$

Since $\left(\varphi\left(\Gamma_{n, k}^{\prime}\right) \times \varphi\left(\Gamma_{n, k}^{\prime \prime}\right)\right) \cap\left(\varphi\left(\Gamma_{n, j}^{\prime}\right) \times \varphi\left(\Gamma_{n, j}^{\prime \prime}\right)\right)=\emptyset$ for all $n \geq n_{0}^{s}$ and $k, j \in$ $\left\{1, \ldots, 2^{n}\right\}$ with $k \neq j$, it follows (4.45), (4.46) and (4.47) that

$$
\int_{\Gamma_{n}}|d \eta||d \xi| \geq \sum_{k=1}^{2^{n}} \int_{\varphi\left(\Gamma_{n, k}^{\prime}\right) \times \varphi\left(\Gamma_{n, k}^{\prime \prime}\right)}|d \xi||d \eta| \geq \pi^{2} 2^{-n-2}
$$

for all $n \geq n_{0}^{s}$. Combining (4.43) with (4.48) hence implies that

$$
\int_{\mathbb{S}^{1}} \int_{\mathbb{S}^{1}} \log \left|\varphi^{-1}(\xi)-\varphi^{-1}(\eta)\right|^{-1}|d \eta||d \xi| \gtrsim \sum_{n=n_{0}}^{+\infty} \frac{j_{n}}{2^{n}} \approx \sum_{n=n_{0}}^{+\infty} \frac{2^{\frac{n}{s}}}{2^{n}}=+\infty .
$$


Therefore (4.42) is complete.

For any $n \geq n_{0}$ and $j_{n}<j \leq j_{n+1}$, by (4.36), (4.34), (4.11) and Jensen's inequality we have that

$$
\begin{aligned}
\sum_{k=1}^{2^{j}} \ell\left(\varphi\left(\Gamma_{j, k}\right)\right)^{p} & =2 \pi \sum_{k=1}^{2^{j}} \mathcal{L}^{1}\left(g_{s}\left(\left[(k-1) 2^{-j}, k 2^{-j}\right]\right)\right)^{p} \\
& \lesssim \sum_{k=1}^{2^{j}}\left(f_{s}\left(k 2^{-j}\right)-f_{s}\left((k-1) 2^{-j}\right)\right)^{p}+\sum_{k=1}^{2^{j}} 2^{-p j} \\
& =2^{(1-p)(n+1)}+2^{(1-p) j}
\end{aligned}
$$

Notice $j_{n+1}-j_{n} \approx 2^{n / s}$ whenever $n \geq n_{0}^{s}$. We then derive from (4.49) that

$$
\begin{aligned}
\sum_{j=j_{n_{0}}+1}^{+\infty} \sum_{k=1}^{2^{j}} \ell\left(\varphi\left(\Gamma_{j, k}\right)\right)^{p} & \lesssim \sum_{n=n_{0}}^{+\infty} \sum_{j_{n}<j \leq j_{n+1}} 2^{(1-p)(n+1)}+\sum_{j=j_{n_{0}}+1}^{+\infty} 2^{(1-p) j} \\
& \approx \sum_{n=n_{0}}^{+\infty} 2^{n\left(1-p+\frac{1}{s}\right)}+\sum_{j=j_{n_{0}}+1}^{+\infty} 2^{(1-p) j}<+\infty .
\end{aligned}
$$

Since $\sum_{j=1}^{j_{n_{0}}} \sum_{k=1}^{2^{j}} \ell\left(\varphi\left(\Gamma_{j, k}\right)\right)^{p}$ is finite, it follows from $(4.50)$ that $\mathcal{E}_{1}(p, p-2,0, \varphi)<$ $+\infty$. Moreover by Lemma 3.3 and Lemma 3.4 we have that $I_{1}(p, p-2,0, P[\varphi])<$ $+\infty$ and $I_{2}(p, p-2,0, P[\varphi])<+\infty$.

Example 4.4. There is a homeomorphism $\varphi: \mathbb{S}^{1} \rightarrow \mathbb{S}^{1}$ such that both $I_{1}(p, \alpha, \lambda, P[\varphi])<$ $+\infty$ and $I_{2}(p, \alpha, \lambda, P[\varphi])<+\infty$ hold for all $p>1, \alpha \in(-1, p-1)$ and $\lambda \in \mathbb{R}$. Moreover for any $p>1$, there is a homeomorphism $\varphi: \mathbb{S}^{1} \rightarrow \mathbb{S}^{1}$ such that $I_{1}(p, \alpha, \lambda, p[\varphi])=\infty$ and $I_{2}(p, \alpha, \lambda, P[\varphi])=\infty$ whenever either $\alpha \in(-1, p-2)$ and $\lambda \in \mathbb{R}$ or $\alpha=p-2$ and $\lambda \in[-1,+\infty)$.

Proof. Take $\varphi: \mathbb{S}^{1} \rightarrow \mathbb{S}^{1}$ as the identity mapping. We have that

$$
\mathcal{E}_{1}(p, \alpha, \lambda, \varphi) \approx \sum_{j=1}^{+\infty} 2^{j(p-2-\alpha)} j^{\lambda} 2^{j}\left(2^{1-j} \pi\right)^{p} \approx \sum_{j=1}^{+\infty} 2^{-j(1+\alpha)} j^{\lambda}<+\infty
$$

whenever $p>1, \alpha \in(-1, p-1)$ and $\lambda \in \mathbb{R}$. Therefore by Lemma 3.3 and Lemma 3.4 both $I_{1}(p, \alpha, \lambda, P[\varphi])$ and $I_{2}(p, \alpha, \lambda, P[\varphi])$ are finite now.

For a given $p>1$, set $j_{n}$ in (4.1) as $\left[e^{2^{n(p-1)}}\right]$. There is $n_{0} \geq 1$ such that (4.2) holds for all $n \geq n_{0}-1$. By following the arguments for (4.5), we have $f$ as in (4.11). Moreover by same arguments as in the proof of Proposition 4.1, there is a constant $C>0$ depending only on $p$ such that

$$
|f(x)-f(y)| \log ^{\frac{1}{p-1}} \log \left(|x-y|^{-1}\right) \leq C
$$


for all $x, y \in[0,1]$ with $x \neq y$. As in (4.36), we finally obtain a homeomorphism $\varphi: \mathbb{S}^{1} \rightarrow \mathbb{S}^{1}$. For any $n \geq n_{0}$ and $j_{n}<j \leq j_{n+1}$, by analogous arguments for (4.41) we have that

$$
\sum_{k=1}^{2^{j}} \ell\left(\varphi\left(\Gamma_{j, k}\right)\right)^{p} \gtrsim 2^{n(1-p)} .
$$

Notice that $\sum_{j_{n}<j \leq j_{n+1}} j^{-1} \approx \log j_{n+1}-\log j_{n} \gtrsim 2^{n(p-1)}$ for all $n \geq n_{0}$. For any $\lambda \in[-1,+\infty)$ it then follows from (4.51) that

$$
\begin{aligned}
\mathcal{E}_{1}(p, p-2, \lambda, \varphi) & \geq \sum_{j=1}^{+\infty} \sum_{k=1}^{2^{j}} \ell\left(\varphi\left(\Gamma_{j, k}\right)\right)^{p} j^{-1} \geq \sum_{n=n_{0}}^{+\infty} \sum_{j_{n}<j \leq j_{n+1}} j^{-1} \sum_{k=1}^{2^{j}} \ell\left(\varphi\left(\Gamma_{j, k}\right)\right)^{p} \\
& \gtrsim \sum_{n=n_{0}}^{+\infty} 2^{n(p-1)} \cdot 2^{n(1-p)}=+\infty .
\end{aligned}
$$

For any $\alpha \in(-1, p-2)$ and $\lambda \in \mathbb{R}$, we have that $2^{j(p-2-\alpha)} j^{\lambda} \gtrsim j^{-1}$ whenever $j \gg 1$. Without loss of generality, we assume that $2^{j(p-2-\alpha)} j^{\lambda} \gtrsim j^{-1}$ for all $n \geq n_{0}$ and $j_{n}<j \leq j_{n+1}$. Hence from (4.52) we have that

$$
\begin{aligned}
\mathcal{E}_{1}(p, \alpha, \lambda, \varphi) & \geq \sum_{n=n_{0}}^{+\infty} \sum_{j_{n}<j \leq j_{n+1}} \sum_{k=1}^{2^{j}} \ell\left(\varphi\left(\Gamma_{j, k}\right)\right)^{p 2^{j(p-2-\alpha)} j^{\lambda}} \\
& \gtrsim \sum_{n=n_{0}}^{+\infty} \sum_{j_{n}<j \leq j_{n+1}} \frac{1}{j} \sum_{k=1}^{2^{j}} \ell\left(\varphi\left(\Gamma_{j, k}\right)\right)^{p}=+\infty
\end{aligned}
$$

for all $\alpha \in(-1, p-2)$ and $\lambda \in \mathbb{R}$. By Lemma 3.2, Lemma 3.3 and Lemma 3.4, we conclude from (4.52) and (4.53) that for any $p>1$ there is a homeomorphism $\varphi: \mathbb{S}^{1} \rightarrow \mathbb{S}^{1}$ such that $I_{1}(p, \alpha, \lambda, P[\varphi])=\infty$ and $I_{2}(p, \alpha, \lambda, P[\varphi])=\infty$ whenever either $\alpha \in(-1, p-2)$ and $\lambda \in \mathbb{R}$ or $\alpha=p-2$ and $\lambda \in[-1,+\infty)$.

\section{Acknowledgment}

The author has been supported by China Scholarship Council (project No. 201706340060) and by National Natural Science Foundation of China (project No. 11571333). This paper is a part of the author's doctoral thesis. The author thanks his advisor Professor Pekka Koskela for posing this question and for valuable discussions. 


\section{References}

[1] K. Astala, T. Iwaniec, G. J. Martin, and J. Onninen: Extremal mappings of finite distortion, Proc. London Math. Soc. 91 (2005), no. 3, 655-702.

[2] O. J. Broch: Extension of internally bilipschitz maps in John disks. Ann. Acad. Sci. Fenn. Math. Volumen 31, (2006), 13-31.

[3] G. Choquet: Sur un type de transformation analytique généralisant la représentation conforme et définie au moyen de fonctions harmoniques, Bull. Sci. Math. (2) 69 (1945) 156-165

[4] R. R. Coifman, and C. Fefferman: Weighted norm inequalities for maximal functions and singular integrals. Studia Math. 51 (1974), 241-250.

[5] T. Iwaniec, G. J. Martin, and C. Sbordone: $L^{p}$-integrability \& weak type $L^{2}$-estimates for the gradient of harmonic mappings of $\mathbb{D}$. Discrete Contin. Dyn. Syst. Ser. B 11 (2009), no. 1, 145-152.

[6] P. W. Jones: Factorization of $A_{p}$ weights. Ann. of Math. (2) 111 (1980), no. $3,511-530$.

[7] H. Kneser: Lösung der Aufgabe 41, Jahresber. Deutsch. Math.-Verein. 35 (1926), 123-124.

[8] V. Kokilashvili, and M. Krbec: Weighted inequalities in Lorentz and Orlicz spaces. World Scientific Publishing Co., Inc., River Edge, NJ, 1991. xii+233 pp.

[9] P. Koskela, Z. Wang, and H. Xu: Controlled diffeomorphic extension of homeomorphisms. Nonlinear Anal. 177 (2018), part B, 586-600.

[10] H. Lewy: On the non-vanishing of the Jacobian in certain one-to-one mappings, Bull. Amer. Math. Soc. 42 (1936) 689-692.

[11] B. Muckenhoupt: Weighted norm inequalities for the Hardy maximal function. Trans. Amer. Math. Soc. 165 (1972), 207-226.

[12] R. Näkki, and J. Väisälä: John disks. Expo. Math. 9, (1991), 9-43.

[13] T. Radó: Aufgabe 41, Jahresber. Deutsch. Math.-Verein. 35 (1926) 49.

[14] G. C. Verchota: Harmonic homeomorphisms of the closed disc to itself need be in $W^{1, p}, p<2$, but not $W^{1,2}$. Proc. Amer. Math. Soc. 135 (2007), no. 3, 891-894. 